\title{
Perspective
}

PERSPECTIVE Actualité en histoire de l'art

$2 \mid 2020$

Danser

\section{Sources iconographiques et chorégraphiques : pour une recherche croisée entre histoire de l'art et études en danse}

Un débat entre Inge Baxmann, Juliet Bellow, Mickaël Bouffard et Lou Forster, mené par Pauline Chevalier et Marie Glon

Inge Baxmann, Juliet Bellow, Mickaël Bouffard, Pauline Chevalier, Lou Forster et Marie Glon

Traducteur : Michèle Veubret

\section{(2) OpenEdition}

Journals

Édition électronique

URL : https://journals.openedition.org/perspective/20047

DOI : 10.4000/perspective.20047

ISSN : 2269-7721

Éditeur

Institut national d'histoire de l'art

Édition imprimée

Date de publication : 30 décembre 2020

Pagination : $51-72$

ISBN : 978-2-917902-90-5

ISSN : $1777-7852$

Référence électronique

Inge Baxmann, Juliet Bellow, Mickaël Bouffard, Pauline Chevalier, Lou Forster et Marie Glon, « Sources iconographiques et chorégraphiques : pour une recherche croisée entre histoire de l'art et études en danse », Perspective [En ligne], 2 | 2020, mis en ligne le 30 juin 2021, consulté le 29 juillet 2022. URL : http://journals.openedition.org/perspective/20047 ; DOI : https://doi.org/10.4000/perspective.20047 


\title{
Sources iconographiques et chorégraphiques : pour une recherche croisée entre histoire de l'art et études en danse
}

\author{
Un débat entre Inge Baxmann, Juliet Bellow, \\ Mickaël Bouffard et Lou Forster, \\ mené par Pauline Chevalier et Marie Glon
}

En 2018, l'Institut national d'histoire de l'art initiait un programme de recherche ${ }^{1}$ sur les pratiques graphiques en danse - gravures, dessins, notations, carnets de chorégraphes dont l'ambition est de construire un champ de recherche commun entre histoire de l'art et histoire de la danse. Alors que les chercheurs travaillant entre danse et arts visuels se sont principalement intéressés aux collaborations entre artistes, danseurs et chorégraphes ou aux pratiques contemporaines hybrides, comment penser aujourd'hui la possibilité d'un partage des corpus et des méthodes entre recherche en danse et en histoire de l'art?

Comment le corps informe-t-il le regard et comment les outils de la recherche en danse peuvent-ils bénéficier à l'histoire de l'art ? Laurence Louppe, dont les travaux ont jeté des bases pour la recherche en danse en France et accordé une large place aux arts visuels, appelait de ses vœux une analyse accrue des images et œuvra pendant plusieurs décennies aux rapprochements entre deux disciplines qui, si elles ne partagent pas la même histoire dans leur construction académique, n'ont eu de cesse de se frôler, de se côtoyer, sans pour autant embrasser leurs impensés communs : les images sous-jacentes, survivantes, dans la création chorégraphique ${ }^{2}$; l'image comme document d'une histoire visuelle de la danse ; des processus graphiques et plastiques qui jalonnent la création chorégraphique : autant de sources qui fournissent des terrains de recherche partagés et communs. Il ne s'agit pas de détailler ici la multitude des points de recoupement entre nos disciplines, mais plutôt de saisir la nécessité de les appréhender ensemble.

Les fonds conservés au Centre national de la danse, à la Bibliothèque nationale de France et à l'INHA ont constitué les premiers supports de ces approches croisées dans le cadre du programme de recherche "Chorégraphies. Écriture et dessin, signe et image dans les processus de création et de transmission chorégraphiques (XVe-XXI ${ }^{\mathrm{e}}$ siècles) ". Le CND représente certainement un gisement de sources insoupçonnées pour les historiens de l'art contemporain : carnets de chorégraphes, partitions, dessins de spectateurs, œuvres réalisées en collaboration entre danseurs et artistes, photographies. Ancrés dans l'histoire de la danse, ces fonds appellent un travail commun que nous avons tenté de mettre en place, par exemple autour du fonds de la chorégraphe Janine Solane ${ }^{3}$ 
qui laisse pour chacune de ses chorégraphies de grands carnets dessinés, composés de multiples calques superposés (fig. 1). Exercices de création et de notation, exécutés en amont du travail en studio avec les danseuses, ces objets-frontières témoignent également d'une fascination de l'artiste pour le dessin et pour la ligne musicale. Celle qui invitait ses interprètes à fréquenter le musée du Louvre, à dessiner régulièrement, faisait un usage du dessin comme d'un outil intermédiaire entre la musique et le geste. Les qualités du trait disent autant de son écoute de la musique que de sa pensée des mouvements dans l'espace.

Au-delà de cette étude de fonds témoignant des pratiques graphiques des danseurs eux-mêmes, il s'agit d'aborder le rôle des images dans la recherche en danse, et la place de la danse dans la recherche en histoire de l'art. Francis Haskell interrogeait ainsi le rôle des images pour l'historien : « la photographie a eu pour l'historien l'effet paradoxal de renforcer l'autorité de toutes les images, même celles qui sont fort antérieures à son invention ${ }^{4}$ ". Ce constat pourrait sans doute être plus marqué encore dans le cas de l'historiographie de la danse : les ouvrages d'histoire de la danse se donnent bien souvent à voir autant qu'à lire, tant ils abondent de reproductions (photographies, gravures et œuvres picturales principalement). Ces dernières sont généralement choisies uniquement pour leur impact visuel - or, bien souvent, elles sont implicitement proposées aux lecteurs et lectrices comme des illustrations du texte, voire comme des aperçus des pratiques dansées (on imagine ainsi volontiers Marie Taglioni dansant pieds nus, comme sur les représentations d'elle en sylphide ${ }^{5}$ ). Les conditions de production de ces images, les conventions et les recherches plastiques dont elles témoignent - et leur lien parfois très éloigné avec la réalité, dont on laisse pourtant supposer qu'elles la donnent à voir de façon transparente - sont rarement interrogés.

À l'inverse, d'autres chercheurs et chercheuses en danse se méfient des images au point de ne pas les inclure dans leurs corpus d'étude (ce qui peut aussi s'expliquer par la nécessité, pour une discipline universitaire reconnue depuis peu, d'affirmer l'existence d'un domaine propre avant d'embrasser des corpus déjà marqués par des approches et des outils venus d'un autre domaine scientifique). Les collections iconographiques peuvent-elles, et à quelles conditions, devenir des sources pour les recherches en danse ? Il y a là aussi un enjeu de taille - et de nature à proposer de nouvelles ambitions

1. Janine Solane, notations chorégraphiques pour La Symphonie Pastorale (1945), Pantin Centre national de la danse, fonds Janine Solane.

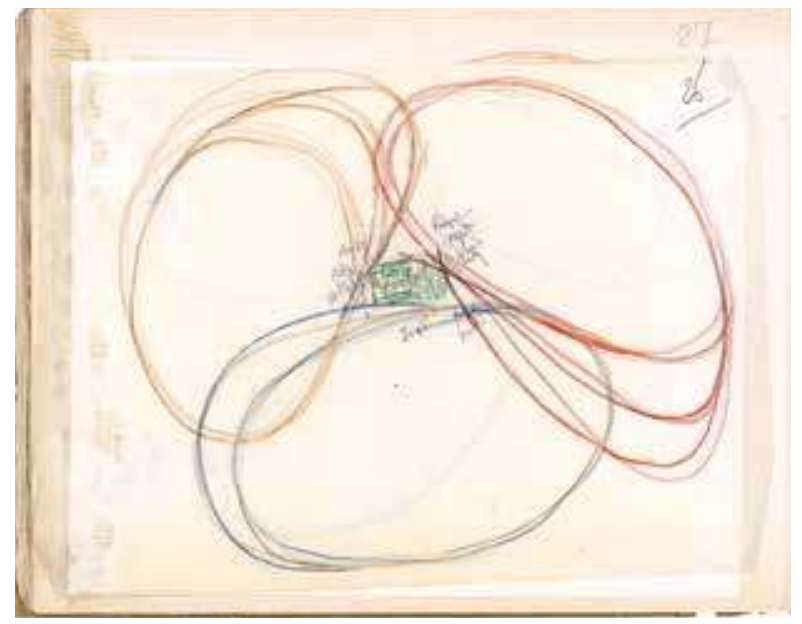
aux programmes, nombreux, impliquant " la danse au musée ". C'est une autre des questions explorées par le programme "Chorégraphies..." de l'INHA : en quoi les sources chorégraphiques peuvent-elles témoigner d'une culture artistique et visuelle, voire d'une pratique inhérente à la création chorégraphique ? Un autre atelier mené dans le cadre du programme, unissant chercheurs en danse et en histoire de l'art, a apporté quelques réponses à ces questions, à partir d'un fonds méconnu et peu étudié de la bibliothèque-musée de l'Opéra : André Jean Jacques Deshayes (1777-1846), danseur et maître de ballet à l'Opéra et au King's Theatre de Londres ${ }^{6}$, semble avoir développé une pratique exceptionnelle du dessin à l'aube 
du romantisme. Si le fonds offre quelques difficultés majeures d'attribution (plusieurs mains, dont celle de Charles Didelot, ont été identifiées), la présence de très nombreuses esquisses de tableaux scéniques (fig. 2) dans un fonds contemporain de Charles Blasis qui invitait tout danseur à " se dessiner ", au propre comme au figuré, incite, là encore, à travailler en commun. La lecture des figures, des notations et des corps suggérés par le crayon passe à la fois par une quête des références iconographiques - la traque des indices rappelant ce connoisseurship que Blasis appréciait justement ${ }^{7}$ - et par une analyse du mouvement, des poses, des tensions et des instants. Au-delà de l'anatomie des

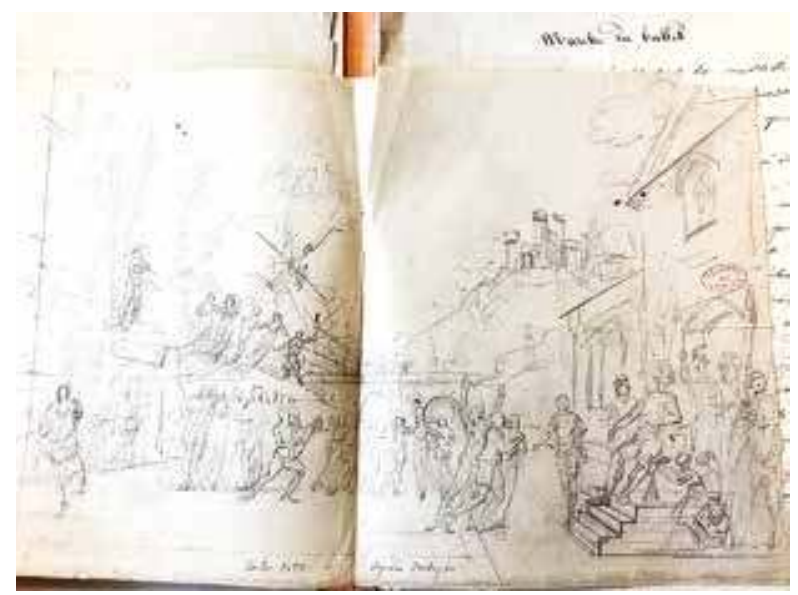

2. Croquis chorégraphique issu du fonds André Jean-Jacques Deshayes (dossier 6), Paris, Bibliothèque nationale de France, bibliothèque-musée de l'Opéra. corps, la lecture des images passe par la connaissance d'une esthétique chorégraphique du début du XIX ${ }^{e}$ siècle, nourrie d'une culture picturale néo-classique et dont la pensée de l'image mérite une approche croisée entre histoire de l'art et histoire de la danse. Nous ne reprendrons pas ici les différentes étapes du programme de recherche, mais ce débat ouvre la discussion sur les potentialités de construction de ce champ commun permettant aussi d'inviter à un renouvellement des objets ${ }^{8}$ et des méthodes.

Sur l'aspect méthodologique, l'un des points principaux repose certainement sur le fait que la recherche en danse est aussi une recherche en acte, qui mêle le geste à l'œil et à l'archive, affirmant le corps comme un outil de savoir - une évidence qui mérite sans doute d'être envisagée dans un champ plus large que celui des études chorégraphiques.

Être danseur, c'est choisir le corps et le mouvement du corps comme champ de relation avec le monde, comme instrument de savoir, de pensée et d'expression. C'est également faire confiance au caractère " lyrique " de l'organique, sans pour autant se référer à une esthétique ni à une mise en forme précise : le geste ou l'état de corps neutre (volontairement désaccentué et travaillant sur l'absence de "dessin") a sa propre qualité lyrique, tout autant que le geste tensionnel spatialité et musicalité. Le tout étant de travailler d'abord aux conditions organiques de cette émergence poétique. Option féconde, le corps devient un formidable outil de connaissance et de sensation?.

Georges Didi-Huberman est revenu à plusieurs reprises sur cette relation de la danse aux Beaux-Arts dans la généalogie du Pathosformel d'Aby Warburg, cette " notion élaborée en grande partie pour rendre compte de cette intensité chorégraphique qui traverse toute la peinture de la Renaissance ${ }^{10}$ ». Déjà présent chez Friedrich Nietzsche et Jacob Burckhardt, ce désir de rapprochement des " arts isolés " s'exprime de façon sous-jacente chez August Schmarsow ou chez Bernard Berenson dont la théorie des " valeurs tactiles " est reprise par Henri Focillon, qui le cite dès les premières lignes de l'Éloge de la main. On pense aussi à Jean Starobinski qui parle quant à lui d'" attention motrice $^{11}$ ". Les propos de Berenson sur Pollaiuolo, à propos du Combat d'hommes nus, restent un exemple majeur de ce regard nourri par l'expérience somatique de l'auteur, qui associe ici l'exercice d'écriture à l'appréhension kinesthésique de l'image par celui qui la regarde comme par celui qui le lit : 
D'où vient l'attrait qui nous y ramène pour nous en faire jouir chaque fois davantage ? [...] le secret du plaisir que nous inspirent ces combattants féroces réside en leur pouvoir de nous communiquer du premier coup une vitalité qui exalte prodigieusement la nôtre. [...] Voyez le pied de l'homme abattu s'arc-bouter sur la cuisse de son ennemi et calculez le formidable effort par lequel il le tient en suspens, tandis que l'autre, pivotant sur lui-même sans lâcher la tête à laquelle il se cramponne, ne déploie pas moins de force pour conserver son avantage. Les manifestations de ces pesées et de ces tensions sont si frappantes, que nous croyons en recevoir la répercussion. Nous nous imaginons répéter ces mouvements, y mettre la vigueur qu'ils exigent et, comme il ne nous en coûte rien, nous supputons déjà l'exaltation où nous jetterait notre entrée en scène ${ }^{12}$.

Empathie kinesthésique et métaphore théâtrale : Berenson dévoile un plaisir du corps et d'une sensation fictive du mouvement par sa représentation, ce point de jonction qui nous intéresse tout particulièrement ici. Observer l'œuvre par le geste, c'est aussi comprendre ce que la construction des images dit d'une pensée du corps et du mouvement qui l'anime. Analyser le geste pictural en danseur transpose l'attention, de la représentation vers l'intentionnalité et le moteur du geste, vers une perception du déploiement global, corporel, du geste, même infime. Peut-on envisager de concilier deux conceptions de l'analyse du mouvement, propres à la recherche en danse et à l'histoire de l'art, pour des corpus qui vont bien au-delà d'une iconographie de la danse ? Mimêsis ou dynamique interne du mouvement, ancrage du geste dans un espace orthonormé ou corps déployé dans l'espace qu'il construit : mettre en regard, d'un point de vue diachronique, ces lectures du corps en mouvement éclaire des échos fondamentaux entre théories de l'art et de la danse. On perçoit alors combien Blasis et la peinture néo-classique partagent plus que des références iconographiques : une appréhension du geste déterminé par l'espace et l'image, un anti-maniérisme commun au corps et à l'œuvre. Le débat qui suit, dans lequel plusieurs chercheuses et chercheurs reviennent sur leur propre pratique, vise à proposer quelques pistes pour œuvrer à ce plaisir de l'interdisciplinarité.

[Pauline Chevalier et Marie Glon]

- Pauline Chevalier et Marie Glon. Des outils partagés : entre " herméneutique du regard» et lecture kinésique de l'image, comment penser la porosité des méthodes entre histoire de la danse et histoire de l'art?

- Juliet Bellow. Je voudrais commencer par une remarque sur la formulation de votre question, car je trouve que la métaphore de la " porosité " apporte une alternative utile à la notion d'« interdisciplinarité ». Discuter de la «porosité » des méthodes signifie admettre l'existence de frontières entre l'histoire de la danse et l'histoire de l'art. Chacune des disciplines se livre à l'interprétation avec son propre lot de questions et d'enjeux ; néanmoins, on peut imaginer que ces frontières sont des membranes perméables plutôt que des murailles imprenables. En tant qu'historienne de l'art, je peux donc percevoir plus clairement mes propres biais disciplinaires, mais aussi la manière dont je peux m'approprier les méthodes de I'histoire de la danse, en complément de celles offertes par l'histoire de l'art, puis m'intéresser aux nouvelles perspectives que l'histoire de l'art peut offrir en retour.

Je vois aussi dans le terme de "porosité » un parfait descripteur de la manière dont les artistes, en Europe et aux États-Unis, ont remis en question les limites du médium au tournant du XXe siècle. Le courant analytique formaliste, qui a dominé l'histoire de l'art jusqu'au milieu du XX⿳亠口冋丁 siècle, a séparé le visuel de l'expérience kinesthésique ou haptique. Pour cette raison, 
ce n'est que récemment que les historiens du modernisme ont commencé à se saisir de toute la gamme des stimuli affectifs et sensoriels que de nombreuses avant-gardes artistiques cherchaient à susciter. Paradoxalement, c'est une historienne de la danse, Gabriele Brandstetter, qui a repéré, dans le passé de l'histoire de l'art, les outils nécessaires pour développer une vision alternative de l'art moderne ${ }^{13}$. Sa " redécouverte » des théories d'Aby Warburg, en particulier son application créative du concept de Pathosformel, a aidé les historiens de l'art à réanimer les images corporelles, depuis longtemps en sommeil, du modernisme.

Je voudrais illustrer ces possibilités avec un exemple spécifique : les peintures simultanées de Sonia et Robert Delaunay dans les années 1910. Leurs abstractions aux couleurs éclatantes ont été traditionnellement appréhendées comme des expérimentations sur la sensation optique de la couleur. Mais, dans ses divers manifestes, Robert Delaunay a relié la perception de la couleur à la sensation de mouvement : son but avoué était de capturer « le mouvement automatiquement synchronisé (la simultanéité) de la lumière ${ }^{14} »$. Pour le couple Delaunay, la manifestation de la couleur mobilisait certes la perception oculaire, mais aussi la sensibilité proprioceptive des individus ${ }^{15}$. Cette sollicitation corporelle s'exprime avec une grande force dans le Bal Bullier de Sonia (1913), une peinture au format panoramique $(0,97 \times 3,90 \mathrm{~m})$ dans laquelle la pulsation de cercles concentriques, évoquant des lumières électriques, se mêle à des arabesques tourbillonnantes qui évoquent des danseurs de tango. En suggérant la tête et les épaules d'une femme vue de dos dans le coin inférieur gauche de la toile, Sonia Delaunay invite les spectateurs et spectatrices à se transporter dans cette fameuse salle de bal et, par une sorte d'empathie méta-kinésique, à ressentir les mouvements dansés dans leur propre musculature.

La reconnaissance des propriétés dansantes de cette peinture rend les frontières qui séparent l'histoire de l'art de celle de la danse encore plus poreuses à nos yeux, d'autant plus que nous pouvons utiliser l'œuvre pour étudier les diverses danses pratiquées à cette époque. Plus concrètement, elle permet de mieux comprendre les dessins exécutés par le couple pour les Ballets russes, en particulier pour Cléopâtre (1909, repris en 1918) : en transférant l'esthétique simultanéiste à la scène, les artistes ont étendu son mouvement implicite à l'espace réel. D'une manière moins directe mais tout aussi importante, les peintures des Delaunay peuvent être exploitées pour étudier les danses populaires et les spectacles dansés de l'époque, du tango aux performances métachoriques de Valentine de Saint-Point.

- Inge Baxmann. La question de l'image dans I'histoire de la danse et dans I'histoire de l'art s'inscrit dans les réflexions actuelles sur les changements de nos cultures de savoir et de nos régimes sensoriels face aux médias numériques. Les expériences sensorielles se trouvent aujourd'hui au cœur de l'analyse culturelle, puisque l'image numérique permet d'être immergé dans une expérience multisensorielle, ce qui modifie notre rapport à l'image et, en conséquence, au monde.

Ainsi, repenser le concept et la pratique de l'imageface au numérique nous renvoie au début de la modernité, à un moment où ils ont été repensés à l'aune de la photographie et du film. Ces nouveaux supports médiatiques ont tous provoqué non seulement une crise de la construction du regard, qui met en relief le rôle du corps dans la perception visuelle, mais aussi une crise des cultures de savoir établies. Dans ce contexte, la reconfiguration du visuel dans la configuration des sens et la remise en question des styles et des codes de perception établis deviennent des tâches de premier plan pour les artistes et les théoriciens de l'art et de la culture de l'époque concernée. Cela constitue bel et bien la base du champ commun du rôle de l'image dans I'histoire de la danse comme dans celle des autres formes d'art.

La crise du régime sensoriel établi qui a marqué la modernité a surtout été induite par les nouvelles technologies médiatiques, qui ont restructuré la perception. Le modernisme 
n'a pas tant consisté en un programme théorique qu'en un processus de transformation des techniques du corps et de la perception collective à partir des nouvelles technologies. Les pratiques de l'image et leur questionnement y ont joué un rôle primordial. D'une part, une étroite relation existait entre la danse et les médias modernes : depuis la fin du XIXe siècle, la danse était le sujet préféré des premiers films. On voulait démontrer ainsi le potentiel du nouveau médium pour capter le mouvement. D'autre part, on observe une profonde méfiance des danseurs modernes vis-à-vis de l'image, qui concernait surtout la visualité dominante de l'époque, et en particulier les codes et les habitudes de perception hérités d'une longue culture livresque. Cette tradition était dominée par une visualité décorporalisée, qui instaurait une distance entre le spectateur et " l'objet » du regard. Les danseurs modernes cherchaient à construire un autre regard, qui puisse surpasser ce dualisme. Mary Wigman, danseuse d'expression (Ausdrucktanz, autrefois traduit par "danse expressionniste »), a ainsi formulé sa version, définitive, de cette attitude critique : "Si vous n'avez pas la vue du danseur, vous n'êtes pas un danseur. » Et cette "vue », bien entendu, peut même se réaliser les yeux fermés. À la base de la critique de l'image par les danseurs modernes se trouve une revalorisation du savoir perceptif du corps. La vision y fait partie intégrante d'une perception multisensorielle que Rudolf von Laban - danseur, chorégraphe et théoricien de la danse moderne - a nommé « sens plastique » ou « sens de la danse » (tänzerischer Sinn), une synthèse des sens qui se réalise dans le mouvement.

- Pauline Chevalier et Marie Glon. La production matérielle des images participe directement d'une histoire des corps en mouvement et d'une histoire de la danse. En quoi ces techniques de production des images (photographiques, filmiques, gravées, etc.) ont-elles aussi contribué à la construction de la postérité de certaines figures marquantes de l'histoire de la danse ? En somme, l'histoire de la danse serait-elle une " histoire de l'art ", marquée par les techniques et les codes de production des images?

- Lou Forster. On pourrait, effectivement, penser qu'une partie non négligeable de l'histoire de la danse est écrite comme un roman de Winfried Georg Sebald. Elle tisse une trame qui lie entre elles une série d'images et en déplie les différentes facettes. Je pense, par exemple, à un passage décisif de l'analyse de Mark Franko dans Dancing Modernism / Performing Politics ${ }^{16}$ où il se penche sur l'expression faciale de Martha Graham à partir d'une photographie de Soichi Sunami. Il y détaille ses lèvres légèrement écartées, ses yeux à moitié clos, afin de cerner les traits formels de son inexpressivité. Son analyse de Lamentation (1930) ne se contente pas de croiser la description des pièces et des sources iconographiques. Les catégories qu'il emprunte à l'analyse des images pour identifier l'absorption, la stase, l'angularité, l'absence de profondeur et de modelé lui permettent de saisir en quoi cette pièce, au croisement du primitivisme et du modernisme, constitue la figure en fétiche. Le récit historique consiste alors autant à écrire sur qu'avec des images.

De très nombreux corpus témoignent de l'importance remarquable des collaborations que nouent celles et ceux qui produisent des représentations des danses et celles avec ceux qui les font. Elle tient probablement à la diversité de leurs usages. On y retrouve aussi bien des enjeux pédagogiques ou réflexifs - quand une ou un chorégraphe, ou une ou un pédagogue cherche à analyser et expliciter les enjeux de sa pratique -, créatifs - quand la fabrique des images et celles des œuvres s'entrecroisent -, documentaire - avec le développement technique, cette préoccupation émerge à la fin des années 1960 -, commerciaux - les compagnies commanditent ou achètent des images pour faire la promotion de leur travail et répondre aux demandes des institutions qui présentent leurs œuvres. Le registre iconographique que j'associe le plus spontanément à la postérité des artistes appartient, 

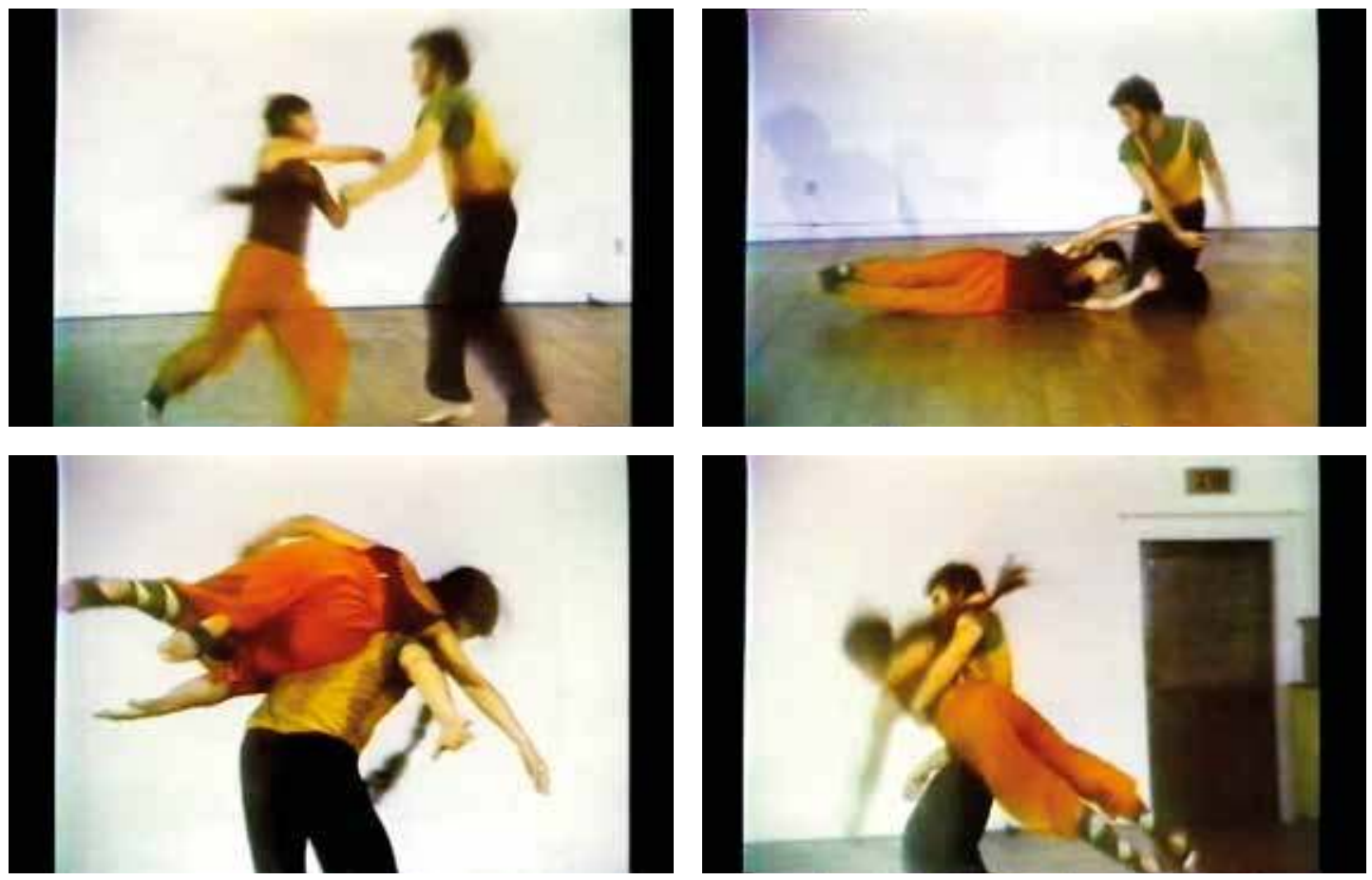

évidemment, à cette dernière catégorie dont on peut multiplier les exemples. Il me semble, toutefois, intéressant d'évoquer un cas plus ambigu ou complexe, comme peut l'être celui de la vidéo de la chorégraphe 3. Lisa Nelson, captures d'écran de Second Glances, 1979 (vidéo et montage: Lisa Nelson ; caméra : Lisa Nelson, Steve Paxton ; interprétation : Alan Ptashek, Nancy Stark Smith). Lisa Nelson intitulée Second Glances ${ }^{17}$ (1979, fig. 3). On l'y voit improviser, avec le chorégraphe Steve Paxton, plusieurs séquences de mouvements caractéristiques du contact improvisation, une technique dont ils sont parmi les principaux promoteurs et qui marque durablement le développement de la danse postmoderne. Les séquences sont montées et mises en boucles de telle sorte que les spectateurs sont amenés à voir plusieurs fois les mêmes mouvements dans différents ordres. En parallèle, la voix monocorde de Nelson décrit les opérations effectuées sur l'image. Le regard en arrière que pose la vidéo permet de mettre en évidence ce qui échappe à la vision instantanée du mouvement. II constitue les prémisses d'un ensemble d'outils de composition chorégraphique comme le Shoot / Talk et les Tuning Scores, qui incorporent l'usage de la caméra et les opérations de montage à la production du mouvement. Second Glances constitue ainsi un document essentiel où se lient la production technique des images et de la danse, tout en permettant $d^{\prime}$ instaurer une relation particulière entre le spectateur ou la spectatrice, et le danseur ou la danseuse. Cette vidéo pose, enfin, un problème générique intéressant, puisqu'elle peut être décrite à la fois comme une œuvre et un document technique en direction des praticiens et des praticiennes du contact improvisation.

- Mickaël Bouffard. La question du potentiel heuristique des images de la danse a connu un tournant décisif dans la littérature en 1987, lorsque Sharon Fermor a mis en évidence les écueils guettant les praticiens de la danse Renaissance qui prêtaient une valeur quasi photographique aux gravures, aux dessins et aux peintures représentant la figure dansante ${ }^{18}$. 


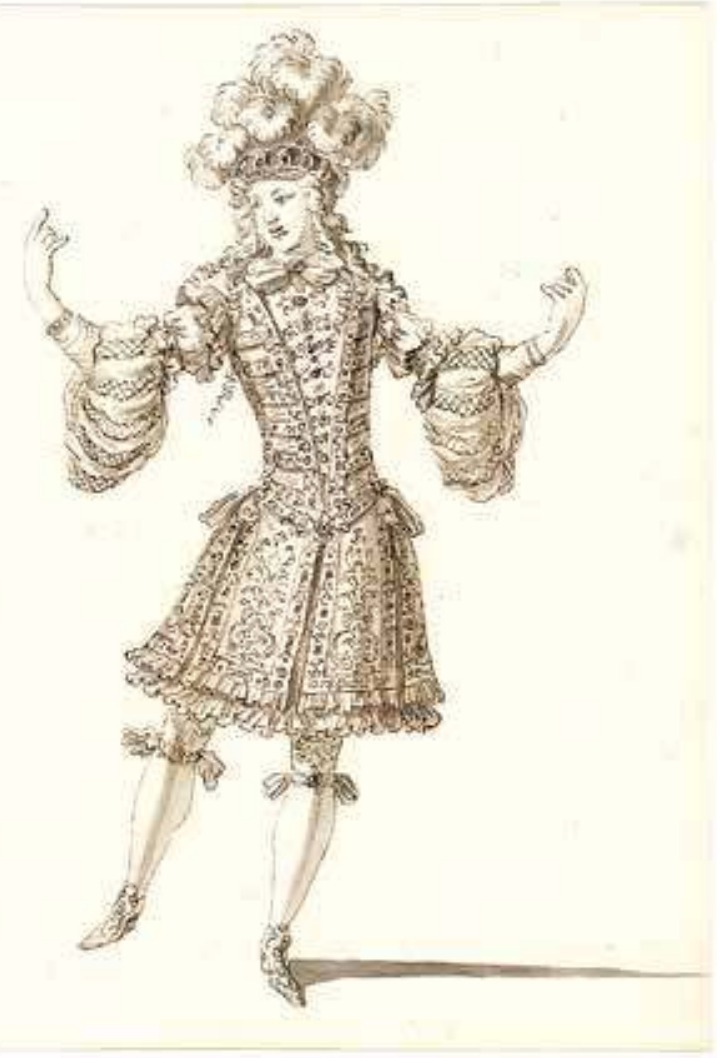

4. Jean I Berain, costume de Carien pour le Triomphe de l'Amour de Lully, vers 1681, plume, encre et lavis gris sur une silhouette à l'eau-forte, Paris, musée du Louvre, collection Edmond de Rothschild.
Cet avertissement nécessaire contre la naïveté de certains danseurs, mais aussi contre celle de plusieurs chercheurs depuis le XIXe siècle, a néanmoins eu un effet rédhibitoire. La revue Dance Research, par exemple, n'a publié aucune étude iconographique sur la danse ancienne pendant près de trois décennies après la parution de cet article. De même, dans les stages et les cours de danses anciennes, une discussion sur la représentation du danseur est très rapidement avortée par ce stérile lieu commun : «Attention, ces images ne sont pas des photos", et sont donc inexploitables, comme si la photographie ne portait pas elle-même son lot de distorsions.

Avec le temps, la mise en garde contre une lecture naïve des images s'est transformée en défiance, incitant à les considérer d'emblée comme de faux témoignages, phénomène que connaît aussi I'histoire du théâtre vis-à-vis des frontispices de pièces imprimées. Or cette attitude revient à refuser de prendre en compte les mécanismes d'encodage de l'image, mais aussi la capacité des artistes à observer le monde qui les entoure et à s'en imprégner. Le plus étonnant est qu'un ouvrage aussi influent que L'œil du Quattrocento de Michael Baxandall ${ }^{19}$ - qui avait bien montré les interactions subtiles à l'œuvre entre l'expérience de la danse et les arts visuels - n'ait pas réussi à contrebalancer la précaution méthodologique pourtant indispensable de

Fermor. L'avènement de la photographie a transformé notre regard, nous poussant à décoder les œuvres anciennes à l'aune de l'instantanéité du cliché photographique ${ }^{20}$, une conception totalement étrangère aux processus de composition de l'image des artistes de l'époque moderne, comme l'a montré d'ailleurs l'histoire de l'art sur la question du prétendu réalisme de la peinture hollandaise ${ }^{21}$.

Prenons un exemple concret, celui de Jean Berain, dessinateur des costumes pour les ballets et opéras sous le règne de Louis XIV. On ne sait pas s'il pratiqua la danse lui-même, mais il en fut le témoin privilégié tout au long de sa vie. Le mémoire du traiteur Cordier, chargé de nourrir les artistes du ballet du Triomphe de l'Amour, compte du pain et du vin pour Berain à toutes les répétitions finales et à toutes les représentations de l'hiver $1681^{22}$. Son expérience, en tant que spectateur, avait fait entrer la belle danse dans son œil, et par voie de conséquence, dans ses doigts. Le costume de Carien pour ce ballet (fig. 4) nous montre le double pli des coudes, un port de bras décrit dans les traités de danse entre 1700 et 1725 . Ce modèle de costume, dessiné avant la représentation du ballet, ne reflète donc pas la réalité du Triomphe de l'Amour, mais représente avec une exactitude technique (par exemple, la main qui ne passe pas la ligne des yeux) une pratique de la danse qui n'est décrite dans les textes que vingt ans plus tard. Berain n'a pas déniché cette posture 
dans une boule de cristal, mais bien dans les usages de son temps. Il choisit donc d'étudier son costume en le posant sur une posture susceptible d'être employée dans le ballet (notamment pour étudier son tombé, ses proportions, etc.), mais aussi pour séduire les commanditaires de ces dessins qui étaient eux-mêmes des connaisseurs de l'art de la danse, sensibles à la justesse et à la grâce des attitudes.

- Inge Baxmann. Tout comme les avant-gardes artistiques modernes du début du XXe siècle, la danse moderne a contribué à l'avènement de nouveaux modèles de visualité. On oublie souvent que le modèle de l'espace développé par Laban doit aussi beaucoup aux découvertes de la microphotographie et du microfilm du début du siècle. Laban se réfère même directement aux travaux de Heinrich Raoul Francé, spécialiste de microphotographie, qu'il cite dans son livre Die Welt des Tänzers de 1920. Le modernisme correspond à un moment où l'unité du monde, ses liens intérieurs sont mis à nu. Les technologies modernes rendent visible une structure profonde, à savoir les principes de construction communs et les structures de base qui relient tous les domaines, de la nature à la technologie et aux arts. Dès la fin du $X I X X^{e}$ siècle, la microphotographie et le microfilm font surgir un monde fait de mouvements, de rythmes, de tensions imperceptibles à l'œil nu mais qui pourtant forment des structures récurrentes dans toute la nature. À partir des images produites par ses technologies se déploie un monde de mouvement, de tension rythmique à la base de modèles d'ordre dans la nature, qui influença profondément le modèle spatial de Laban, dont l'icosaèdre, conçu à partir du cristal, est un bon exemple. Selon Laban, le mouvement est la forme privilégiée pour apercevoir un environnement qui se compose principalement de tension rythmique. Il se révèle donc d'abord par la " perception du danseur ». Une telle perception multi-perspectiviste, qui active tous les sens, fonctionne intuitivement.

La critique des danseurs vis-à-vis de la visualité dominante du début du XXe siècle correspond à la critique de l'image portée par les avant-gardes dans le contexte de la Neues Sehen («nouvelle vision »), développée par les artistes autour du Bauhaus. Leur critique d'une vision décorporalisée est proche de celle de Laban, qui avait suivi des cours de peinture avec Paul Klee et Vassily Kandinsky. L'objectif de l'avant-garde moderne était déjà de dissoudre la séparation entre l'image et le spectateur et de faire de l'acte de voir une expérience plurisensuelle. En étant immergé dans l'œuvre d'art, le spectateur devait expérimenter de nouvelles perceptions (par exemple, des analogies ou des correspondances inattendues) qui modifiraient sa vision du monde. Pour ce faire, il fallait le "déshabituer » d'une manière de voir qui cherche d'abord le « contenu » de l'image, et le sensibiliser à la dynamique des formes, des couleurs et des structures, afin de lui permettre de percevoir I'image à travers tous ses sens.

Le photographe Laszlo Moholy-Nagy chercha quant à lui à découvrir le potentiel de l'image photographique pour organiser des perceptions nouvelles et, ce faisant, révolutionner l'image du monde. Il avait inclus notamment la photographie végétale dans son livre Malerei, Fotografie, Film, publié en $1927^{23}$ (fig. 5). Lecteur passionné d'Heinrich Raoul Francé, il s'approprie sa thèse d'une équivalence structurelle des formes de la nature et de la technologie, et l'étend à l'art. En conséquence, l'art acquiert le rôle primordial de médiateur entre la vie et la technologie. Il fallait ainsi libérer les technologies des médias d'une relation de stricte mimêsis avec le réel, afin de libérer leur dimension artistique, et améliorer les capacités sensuelles de l'être humain moderne. Comme pour Klee, Kandinsky et Oskar Schlemmer au sein du Bauhaus, pour lui, l'art a pour tâche d'entreprendre une formation perceptuelle continue afin de sensibiliser I'humanité à l'expérience du monde moderne. Dans sa recherche d'une nouvelle culture du sensible, tout le Bauhaus s'est appuyé sur la réceptivité de l'organisme humain vis-à-vis de la lumière, de la couleur et du rythme, laquelle nécessite la mise en mouvement du corps qui 


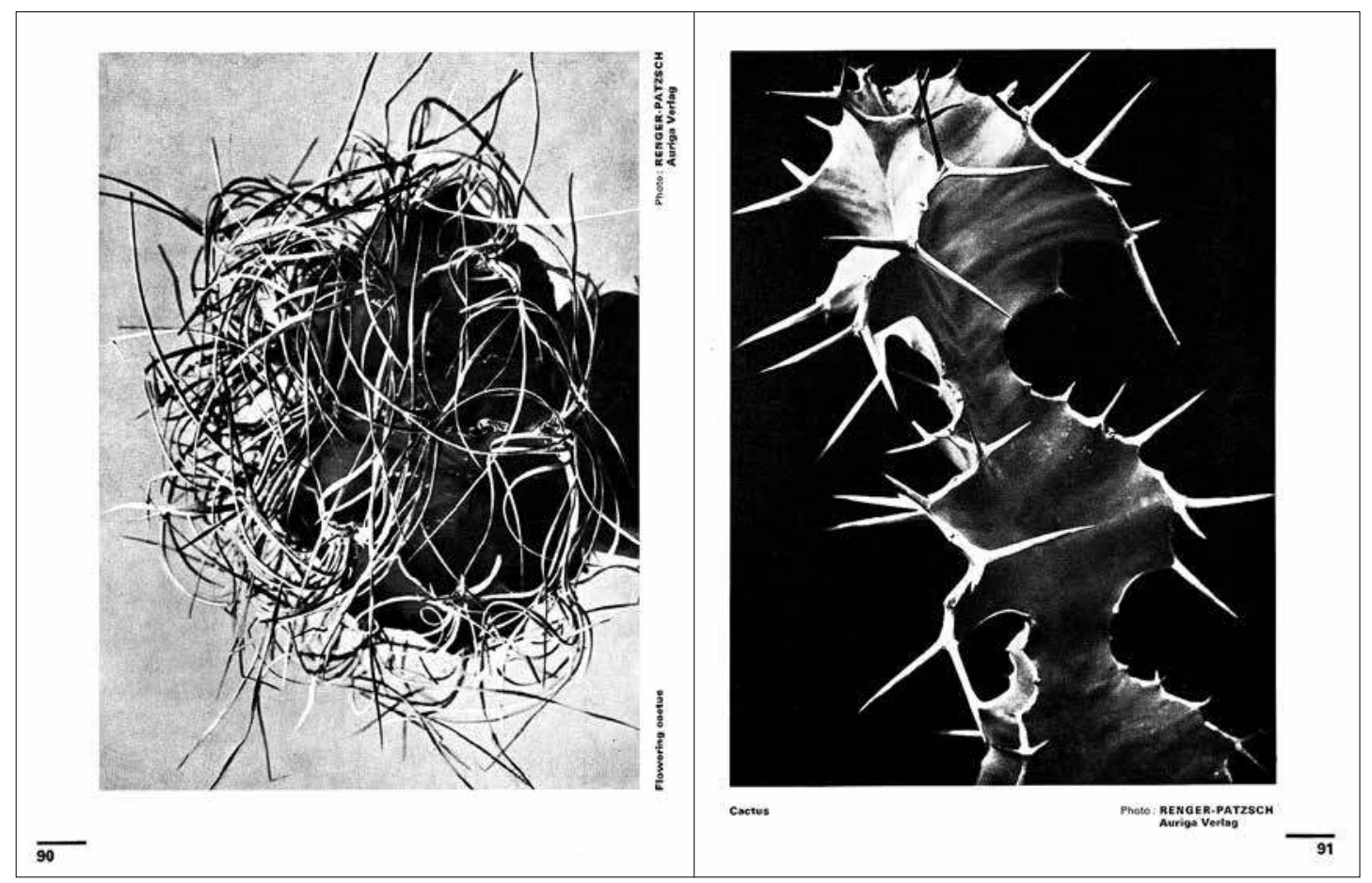

5. Laszlo Moholy-Nagy, Painting, Photography, Film, Londres, Lund Humphries, 1969 (facsimilé de l'édition Malerei, Fotografie, Film, Mainz, Florian Kupferberg Verlag, 1967, tirée de : Bauhausbücher, n 8, Leipzig, Hesse und Becker, 1927), p. 90-91. réagit à l'environnement moderne avec tous ses sens. Leur « art de voir », considéré comme une technique perceptuelle basée sur le lien entre la lumière colorée, le tonus musculaire et, par conséquent, sur un corps rythmiquement en mouvement, constituerait ainsi un « entraînement de la perception », une approche cinétique de l'image avant la lettre.

- Pauline Chevalier et Marie Glon. Plus précisément, pour le cas du XXe siècle, l'iconographie de la danse est aussi le fruit d'un travail dans le studio du photographe (on pense par exemple à l'activité importante des studios dans l'Allemagne des années 1920-1930, mais pas uniquement). Comment l'image de la stase a-t-elle informé celle du mouvement, et comment envisager la « lecture » de la danse par ceux qui ont eu la charge de la saisir ?

- Inge Baxmann. Les danseurs modernes étaient bien conscients du fait que la reconstruction d'une histoire de la danse à partir des images consistait en une « narration avec une différence » et non en une documentation, car les images impliquent des différences de codes, de techniques du corps et d'habitudes de perception relatives à l'époque concernée (et en partie inconscientes), autant d'éléments qui constituent leur historicité immanente. La possibilité d'exister, pour une histoire de la danse, dépend à la fois du potentiel des images à faire sortir la danse - longtemps considérée un art secondaire - des archives de la culture occidentale basée sur l'écriture, et des limites de ces mêmes images pour capter, présenter et construire une narration de la danse, limites liées précisément à leur historicité immanente.

En s'appuyant sur les idées développées par la danse et les avant-gardes du début du siècle précédent, on peut imaginer de travailler à renouveler nos relations aux images d'archive. Si l'archive est principalement une pratique qui se réalise dans le cadre de l'interaction 
des spectateurs avec le matériel historique, le rôle d'une telle médiation - et donc de l'acte archivistique - est de transformer ce matériel d'un monde passé en matériel pour un monde à venir. Ainsi, de nouvelles possibilités de présentation et de narration de l'histoire de la danse, qui permettent d'ancrer les images historiques dans les contextes sensoriels et culturels de leur temps, rendant ces images effectivement lisibles, contribueraient à une compréhension élargie de l'histoire culturelle, intègrant l'histoire du corps et des sens jusqu'aux changements les plus récents de nos modes de perception, d'émotion et d'expérience dans la culture numérique. En sortant l'image de la stase, une telle recontextualisation mobilise le document visuel en l'intégrant et en le confrontant aux questionnements actuels et permettrait de faire naître de nouveaux points de vue sur le matériel historique.

Il est devenu courant, pour les musées, de produire des installations interactives où les images sont présentées de telle façon que les spectateurs soient physiquement intégrés, immergés dans une expérience du matériel historique. Les images de la danse - comme celles des autres arts - contribuent ainsi à construire des visions du monde qui ouvrent la possibilité de faire émerger de nouvelles manières d'être au monde, dans le temps et dans I'histoire. Les médias numériques permettent de présenter le matériel historique de telle sorte que les spectateurs puissent l'expérimenter, et non sur un mode seulement cognitif. L'immersion fait partie de toutes les cultures, mais, depuis la fin du XIXe siècle, le film a créé de nouvelles formes d'immersion, aujourd'hui encore bouleversées par les médias numériques : ces derniers permettent non seulement de faire tourner les images à 360 degrés et de faire l'expérience de la réalité virtuelle, mais ils offrent aussi la possibilité de choisir la forme sous laquelle les données sont présentées. Il est possible, par exemple, de transformer du matériel visuel en son, ou de lier la vision au toucher et à la proprioception. Les images, les technologies des médias et d'autres formes de savoir (comme des témoignages, par exemple), la mémoire du corps de danseurs ou de danseuses, les codes et les habitudes de perception interagissent dans ce processus, dans un enchevêtrement constant entre l'image et les éléments qui forment son contexte.

Pourtant, il n'est pas toujours nécessaire de recourir aux technologies numériques pour proposer des expériences multi-sensorielles : nous les avons déjà incorporées en tant qu'habitudes de perception. C'est probablement cela qui nous fascine dans les performances et installations comme celle de Yoann Bourgeois, La Mécanique de l'Histoire, de 2017 (fig. 6 ; ou comme le film qui en découle, Les Grands Fantômes, 2020). Ce dispositif met en scène une réflexion sur le temps et l'espace qui engage les spectateurs, bien qu'immobiles, dans une visualité multi-perspectiviste, tout simplement en regardant les différentes manières d'explorer le mouvement, l'équilibre et le déséquilibre mis en scène par les acteurs.

Je voudrais enfin ajouter quelques réflexions méthodologiques. Dans une approche interdisciplinaire, ce sont surtout les apports de la neuroscience et de la théorie des médias qui s'avèrent particulièrement intéressants. L'image et sa perception font l'objet de nouveaux modèles de visualité, qui cherchent à explorer le potentiel des médias technologiques pour susciter des effets corporels et reconfigurer le visuel en l'intégrant aux autres sens. Curieusement, ils reprennent la critique d'une conception de l'image menée au début du $\mathrm{XX}$ siècle, aussi bien par les danseurs modernes que par les partisans de la Nouvelle Vision de l'époque. Les neurosciences soulignent que la qualité fondamentalement multi-sensorielle de la perception visuelle : ce processus implique aussi d'autres sens et remet ainsi en question leur ancienne compartimentation.

Le changement actuel de paradigme, que l'on désigne par l'expression embodied cognition approach (une approche dite de la " cognition incarnée »), a des conséquences importantes pour l'image. Il s'agit, à travers ces théories, de comprendre l'esprit comme un ensemble de processus physiques dérivés à la fois du cerveau et du corps d'un individu, à la base de son 


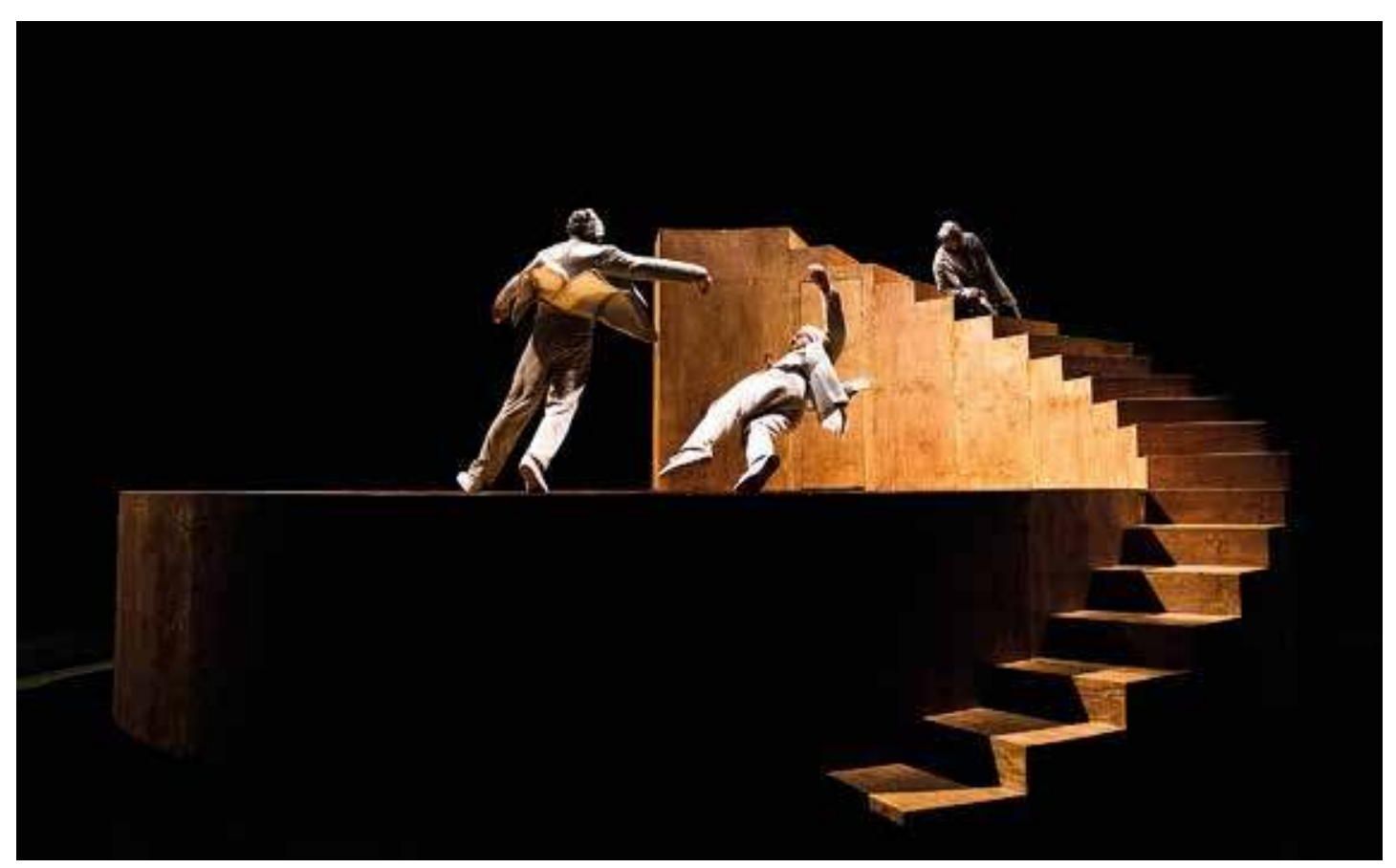

6. Yoann Bourgeois, La Mécanique de l'Histoire, une tentative d'approche d'un point de suspension, Paris, Panthéon, 2017. action dans le monde physique. Cet embodiment est multisensoriel et résulte des effets des signaux visuels, auditifs, tactiles, olfactifs et gustatifs ; c'est un enchevêtrement immersif qui se produit à travers et dans le contexte et l'environnement.

- Pauline Chevalier et Marie Glon. On connaît le rôle des images dans l'élaboration du langage chorégraphique d'Isadora Duncan ou de Vaslav Nijinski, des images à la fois incarnées et incorporées, mais, au-delà de ces exemples bien étudiés, peut-on saisir comment la gravure ou la peinture ont pu laisser une empreinte dans la construction des corps dansants - non seulement dans les emprunts, les citations, parfois aisément saisissables, mais aussi dans la potentialité de la représentation du corps ? Charles Blasis, au début du XIX siècle, invitait les danseurs à "se dessiner ", c'est-à-dire à imaginer la présence d'un artiste ${ }^{24}$ qui saurait saisir la pose. Comment la danse a-t-elle parfois été pensée par les images qu'elle peut potentiellement générer?

- Mickaël Bouffard. Pour comprendre comment la gravure et la peinture ont laissé leur empreinte sur la construction des corps dansants, il faut se plonger dans les méthodes $\mathrm{d}^{\prime}$ incorporation en usage sous l'Ancien Régime. Pour Antoine Coypel, « si les spectacles peuvent être utiles aux peintres; s'ils doivent étudier les gestes des acteurs, des danseurs et des pantomimes : ces derniers peuvent aussi s'instruire sur les grands peintres et sur la beauté de la peinture ${ }^{25}$ ». Au XVIII ${ }^{\mathrm{e}}$ siècle, cette idée que le danseur doit étudier les arts visuels pour construire ses attitudes, pour exprimer correctement les passions ou pour savoir composer des groupes sur scène est partagée par maints maîtres à danser comme John Weaver en Angleterre, Jean-Georges Noverre en France ou Gennaro Magri en Italie. La même recommandation, faite cette fois aux comédiens, s'accompagne d'une méthode d'imprégnation visuelle décrite par le Jésuite Franciscus Lang, qui n'est pas sans rappeler 
les exercices de visualisation ignaciens. L'interprète devait scruter attentivement les détails d'une image pour l'intérioriser puis, une fois sur scène, il devait l'invoquer dans son imagination pour en faire naître dans son corps la manifestation extérieure ${ }^{26}$.

Un siècle avant Blasis, le maître à danser anglais Kellom Tomlinson comparait lui aussi son art à celui du dessinateur (fig. 7) :

Imaginons-nous comme autant d'images vivantes dessinées par les plus excellents maîtres, conçues exquisément de manière à procurer aux spectateurs le plus grand plaisir ; pour ce faire, évidemment, nous devons placer nos corps dans une telle disposition [...] que si nos actions ou postures en venaient à être tracées, elles puissent soutenir l'examen le plus rigoureux des juges les plus critiques ${ }^{27}$.

Cette obsession de la belle image que doit produire le danseur à chaque instant est telle qu'elle pousse Tomlinson à déplorer, quand il décrit la façon convenable de marcher, que le corps ne soit pas dans une posture gracieuse le tiers du temps. En effet, la marche consistant pour lui en l'alternance de la quatrième et de la première positions, le corps se trouve trop souvent en transition entre deux équilibres, dans une situation instable et donc désagréable à l'œil. C'est dans cette optique que sont décrits les pas contenus dans les traités de Raoul-Auger Feuillet ou de Pierre Rameau, à savoir comme des enchaînements fluides (" naturels ", disait-on alors) d'un certain nombre de positions régulières. Ces points de passage doivent donc être exécutés avec exactitude, mais sans trop s'y arrêter, pour éviter que le mouvement ne devienne saccadé et perde de son coulant. Cette double exigence pour la perfection picturale et l'aisance cinétique du danseur met en lumière une conception de la grâce construite par la culture des images.

Une autre méthode, appliquée cette fois à la danse dite en prose (c'est-à-dire le maintien, les gestes, la marche et les révérences, par opposition à la danse dite poétique qui se fait en musique au bal ou au théâtre), va plus loin encore : I'intériorisation par le dessin. Quand Sébastien Leclerc donnait à copier ses élégantes petites figures au jeune duc de Bourgogne, ce dernier devait s'appliquer à bien observer les contours, à considérer l'effet que produit à l'œil l'agencement varié des bras et des jambes. Cette attention, requise par le dessin, est une autre manière de mieux ancrer, par une sorte de diversion, les règles tacites du bon air et de la bonne grâce. La même vertu est attribuée aux planches de Bernard Picart qui proposent à la copie toutes sortes de mains tenant gracieusement des tricornes, des éventails, des jupes, qui représentent des ports de bras ou exemplifient plusieurs manières de disposer les doigts avec art (fig. 8).

- Pauline Chevalier et Marie Glon. À I'inverse, ce n'est pas seulement la danse qui est informée par les images mais aussi les créateurs d'images qui ont été fascinés par la danse et la possibilité, à partir de corps dansants, de développer de

7. Kellom Tomlinson, The Art of Dancing Explained by Reading and Figures, Whereby the Manner of Performing the Steps is Made Easy by a New and Familiar Method... by Kellom Tomlinson, dancing-master..., Londres, Printed for the author, 1735, pl. XV, Londres, British Library.

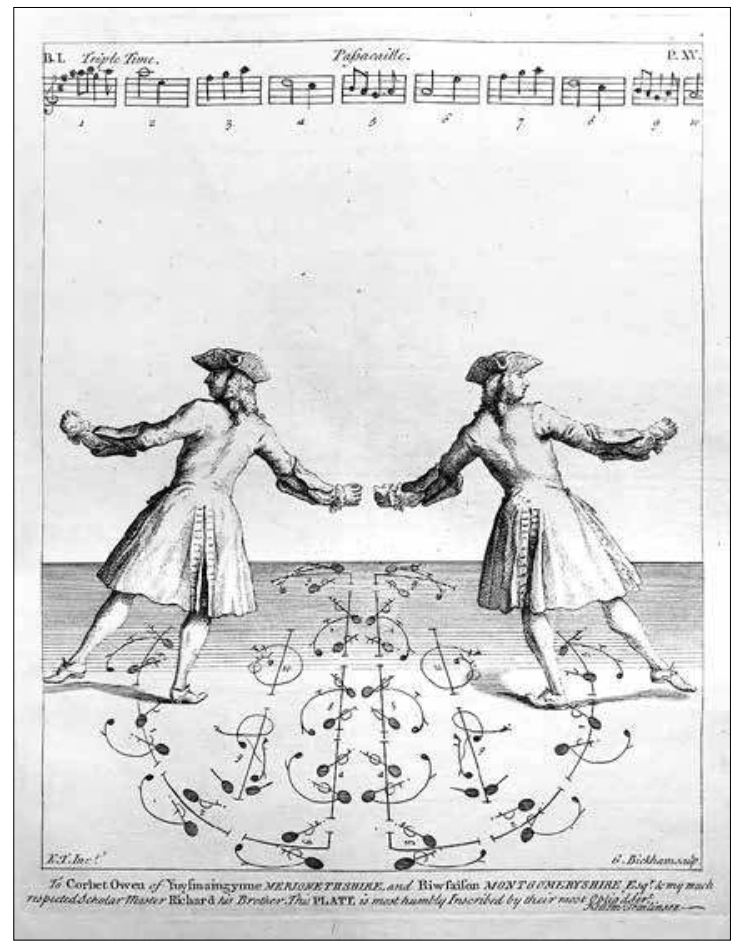




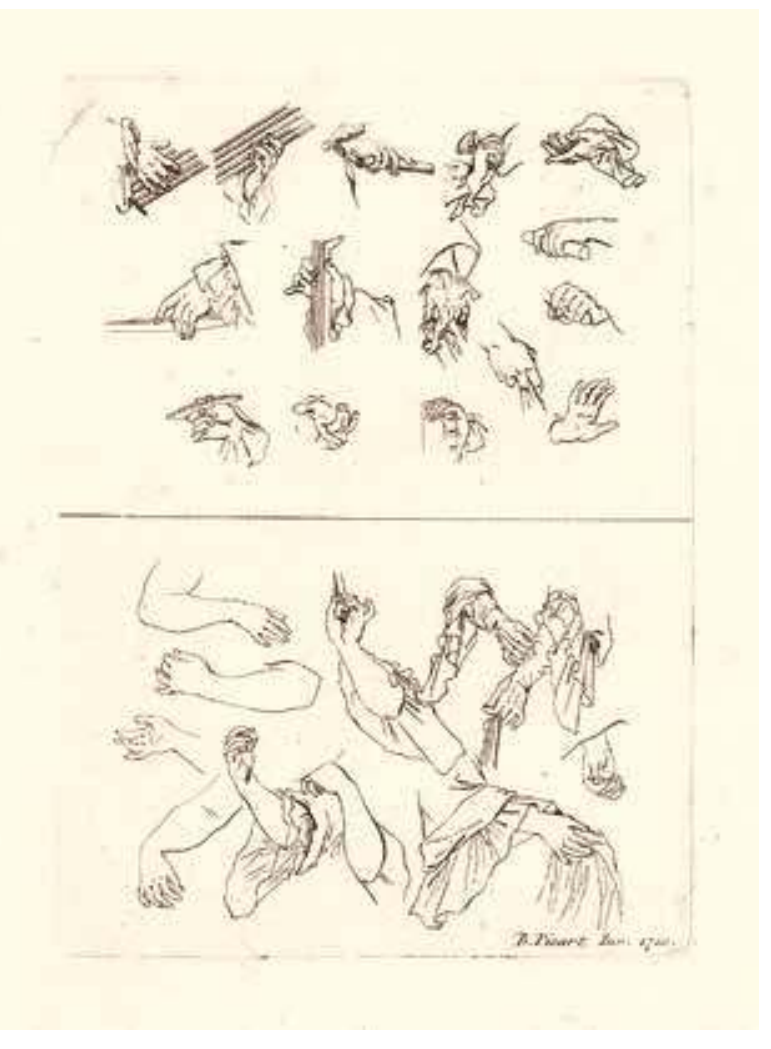

8. D'après Bernard Picart, Modèles de bras et de mains pour apprendre à dessiner (dont un port de bras d'opposition pour la danse), 1710, estampe, Amsterdam, Rijksmuseum. nouvelles formes de représentation ou de mettre leurs techniques en jeu. Peut-on, dans ce cadre, envisager une relation mimétique des gestes? L'implication kinesthésique de l'observation de la danse par l'artiste peutelle générer un mimétisme et une transposition de la qualité d'un geste dansé à un geste pictural?

- Juliet Bellow. À l'aube du Xxe siècle, de nombreux artistes européens ont cherché un appui dans la danse pour contester les conventions et les techniques existantes. Comme Nell Andrew et moi l'avons défendu ailleurs, la danse a occupé une position prédominante dans le développement de la sensibilité moderniste, ouvrant la voie aux autres arts en reconfigurant les conceptions traditionnelles du temps, du mouvement, de la forme et de l'espace ${ }^{28}$. Mais, en vertu du caractère intrinsèquement éphémère de la danse, la plupart des performances qui ont catalysé ces nouveaux répertoires formels n'existent plus en tant que telles. Ainsi, dans de nombreux cas, les représentations des artistes constituent de précieuses « archives d'observation » pour des performances désormais perdues ${ }^{29}$. La relation de ces œuvres aux danses qu'elles visent à représenter n'est évidemment pas directe : nous ne pouvons voir le danseur ou la danseuse qu'au travers des médiations de

l'artiste. Dans le cas des danses dites " exotiques", découvertes par le public européen à cette époque, ce problème était exacerbé par « un déséquilibre des échanges construits par le sexisme et le racisme »; une dynamique qui reflétait, dans un microcosme, des structures de pouvoir géopolitique plus vastes ${ }^{30}$.

Pour prendre un exemple, nous pourrions observer les dessins exécutés par Auguste Rodin du Ballet royal du Cambodge (fig. 9a), une compagnie en visite en France pendant l'été 1906 à l'occasion de l'Exposition coloniale de Marseille ${ }^{31}$. Lors d'une série de poses étalées sur quelques jours, Rodin exécuta d'innombrables croquis de ces danseuses tombées aujourd'hui dans l'anonymat. Dans des interviews accordées à des critiques d'art, Rodin a affirmé qu'il appliquait la technique du « dessin à l'aveugle » : sans quitter les danseuses des yeux tandis qu'elles évoluaient, il esquissait librement sur la feuille. En d'autres termes, il postulait le principe d'un continuum entre le corps dansant perçu par l'œil et la main qui dessinait. En insistant sur la qualité physique de ce processus, Rodin forgeait un lien mimétique entre la danse et le dessin. Cette connexion entre le geste représenté et le déroulement de sa restitution est encore plus radicale dans les dessins des mains des danseuses : I'un d'eux, par exemple, montre le pouce et l'index d'une danseuse réunis en forme de mudra, qui évoque la position d'une main tenant un crayon (fig. 9b). On ne peut pas commenter ces images sans aborder la manière dont elles reflètent et perpétuent l'exercice du pouvoir 
colonial. En exprimant sa profonde affinité avec la danse cambodgienne, Rodin affirmait l'originalité de son style et justifiait son rejet des normes culturelles et esthétiques françaises. D'un autre côté, en utilisant les corps, ethniquement typés, des danseuses cambodgiennes comme terrain d'expérimentation formelle, il affirmait son privilège et son autorité, en tant qu'homme blanc et artiste célèbre. Cependant, il serait injuste de réduire les dessins à cette relation d'inégalité structurelle. Les traits de crayons enchevêtrés et les couches de repentirs nous confirment que leur exécution ne fut pas aussi simple que Rodin le prétendait. Le processus mettait en jeu une série $d^{\prime}$ interactions complexes entre des corps qui partageaient un espace, chacun répondant à la présence et aux actions de l'autre. Et, bien qu'ils soient médiatisés par le filtre des malentendus et des partis-pris de Rodin, ses dessins nous offrent I'un des seuls moyens d'accès à la connaissance de ces quarante-deux jeunes femmes qui ont fait le voyage de Phnom Penh à Paris et à Marseille, pendant l'été 1906.

- Pauline Chevalier et Marie Glon. L'analyse du mouvement développée aujourd'hui dans le champ chorégraphique propose des outils et un vocabulaire qui permettent de saisir avec précision un geste, de « lire ${ }^{32}$ » le mouvement et de le décrire : les concepts fondamentaux de l'analyse du mouvement peuvent-ils nourrir une méthodologie renouvelée en histoire de l'art, mêlant une analyse des sources et des représentations du mouvement? Le concept de " savoir incorporé » vous semble-t-il opérant d'une discipline à l'autre?

9a. Auguste Rodin, Danseuse cambodgienne, juillet 1906, Paris, musée Rodin.

9b. Auguste Rodin, Étude de main de danseuse cambodgienne, juillet 1906, Paris, musée Rodin.
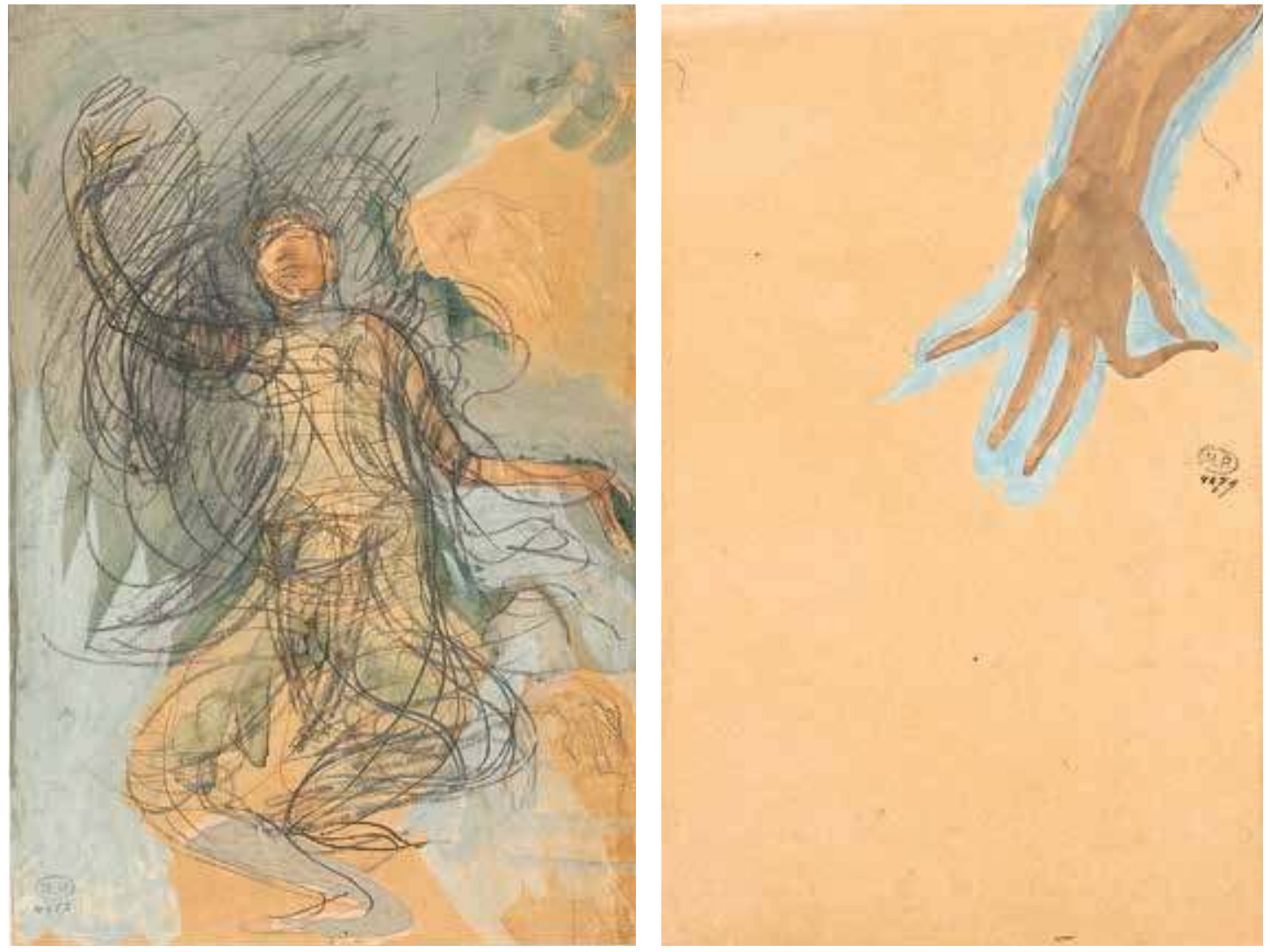

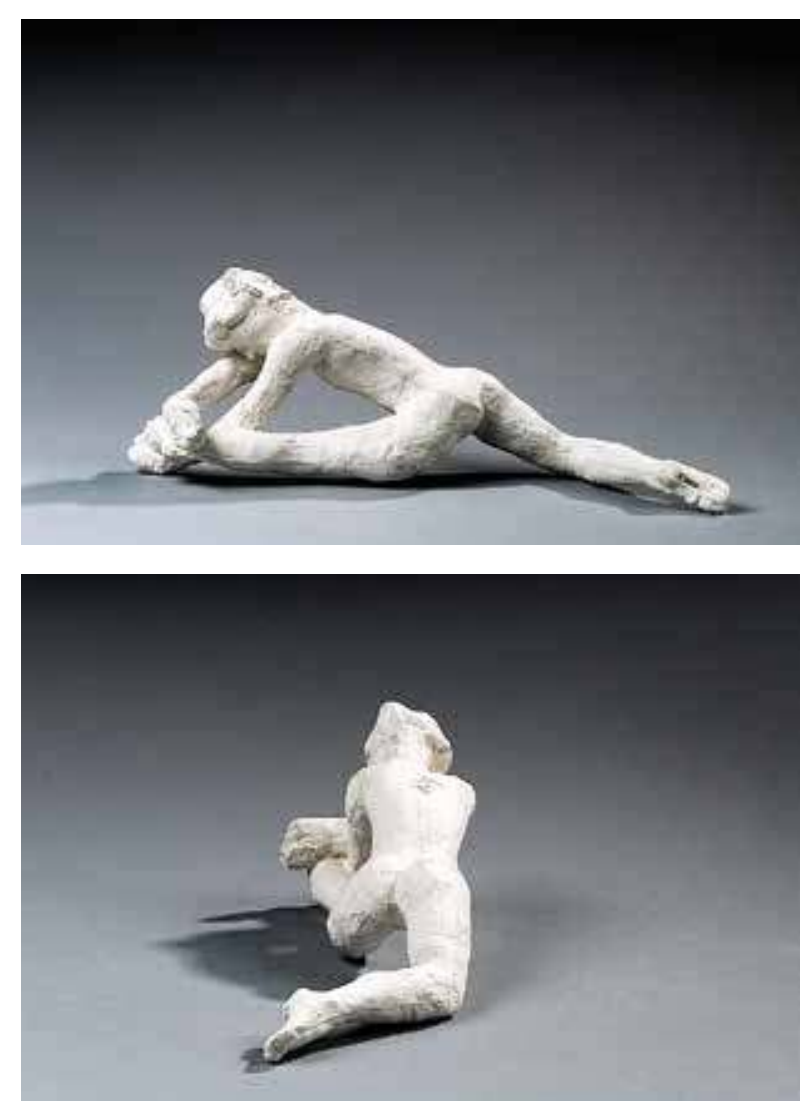

10a-b. Auguste Rodin, un plâtre de la série Mouvement de danse, depuis deux angles de vue différents, Paris, musée Rodin.
- Juliet Bellow. Le savoir incorporé peut faire progresser la recherche en histoire de l'art de différentes façons. Il peut activer nos réponses kinesthésiques aux œuvres d'art ; il peut ranimer des objets inertes; il peut révéler des aspects, restés en sommeil, de certains objets ou certaines pratiques du passé, ou leur attribuer de nouvelles significations. On pourrait trouver un exemple de ces potentialités dans un récent projet de recherche sur la série des Mouvements de danse de Rodin (vers 1911, fig. 10a-b). Ces sculptures de petit format, avec leurs corps déformés et leurs postures invraisemblables d'un point de vue anatomique, ont longtemps dérouté les spécialistes. En 2015, Alexandra Gerstein, conservatrice à la Courtauld Gallery, fut à l'initiative d'une collaboration avec une équipe de chercheurs venus de l'Institute for Movement at the Royal Central School of Speach and Drama, à Londres, pour étudier ces œuvres ${ }^{33}$. Après une observation approfondie des sculptures de Rodin et de ses dessins associés, les chercheurs ont créé leurs propres réponses corporelles à ces compositions. Le but était de comprendre de l'intérieur les postures transcrites par Rodin, en vue d'obtenir de nouveaux aperçus sur leurs sources éventuelles, leurs mécanismes physiques ainsi que sur leur apparence extérieure. Dans plusieurs cas, cette méthode d'incarnation a donné des résultats satisfaisants : certaines compositions, certaines postures considérées comme impénétrables et étranges se sont révélées intelligibles. Il est devenu évident que la plupart des sculptures de cette série n'étaient pas conçues pour se tenir debout, comme elles sont présentées actuellement dans la plupart des musées. En couchant les figures sur le dos ou sur le côté, ou bien en les retournant la tête en bas, leurs poses, tout à coup, faisaient sens.

Cette « libération » des sculptures de leur position verticale figée nous rappelle que peu d'entre elles étaient fixées à un socle du vivant de Rodin. Comme les petites mains, séparées du corps, qu'il créa à peu près à la même époque, il avait conservé ces figures dans un état de mobilité qui lui permettait de modifier continuellement leur position. Comme l'a remarqué de manière mémorable Leo Steinberg, elles sont conçues pour « être cueillies par une main qui les fera tourner entre ses doigts délicats». Pour ressentir la qualité sculpturale d'une telle œuvre, écrit Steinberg, "nous devons la saisir, la faire pivoter et la restituer à ce même médium spatial dans lequel Rodin l'a conçue ${ }^{34} »$. En retirant le corps dansant du socle, Rodin rend radicalement contingentes, non seulement la composition de l'œuvre, mais aussi sa relation à l'environnement et son message au spectateur : chaque fois que l'artiste faisait danser l'une de ces sculptures dans sa main, il exécutait une " performance » différente. 
Un autre projet relié à l'exposition Rodin et la danse (Courtauld Gallery, 2015) élargit les potentialités que le savoir incorporé peut apporter à l'œuvre de Rodin. La chorégraphe Shobana Jeyasingh a créé, spécifiquement pour le lieu, une courte pièce in situ, un solo intitulé Études, pour une danseuse, Noora Kela, qui évoluait dans la galerie à côté des Mouvements de danse. Comme on le voit dans le film de sa performance, ses postures reflètent parfois celles des sculptures - souvent de façon répétitive, car son image est reflétée dans les panneaux de la vitrine où les œuvres sont enfermées. Toutefois, en la voyant évoluer, prendre ces poses et les quitter, en la regardant vaciller, nous comprenons que ses « études » ne sont pas la réplique passive des compositions de Rodin, mais qu'elle les remodèle et les réinvente activement.

- Lou Forster. On pourrait penser que le concept de «savoir incorporé » s'applique particulièrement bien à notre sujet. Les pratiques de danse mettent en jeux l'intégration par les danseurs et les danseuses de techniques, de rythmes, de coordinations, etc. Or cette expérience empirique constitue une ressource fondamentale pour regarder et comprendre non seulement des danses, mais encore leurs représentations, comme vient de l'expliquer Juliet Bellow dans le cas des sculptures de Rodin.

Toutefois, le concept de «savoir incorporé » tel que le déploie Charles Goodwin me semble plus ambitieux encore ${ }^{35}$. Le sociologue a étudié la manière dont le corps intervient dans chacune de nos pratiques de cognition, mêmes celles très éloignées de la danse. II se penche, par exemple, sur une situation de fouille archéologique durant laquelle une professeure et son étudiante cherchent à déterminer les limites de deux strates géologiques à partir d'un nuancier. L'accordage perceptif qui se joue entre ces trois acteurs participe de la production d'une manière de voir qui se trouve au cœur de l'archéologie comme discipline. Ainsi, peu importe l'objet sur lequel porte la connaissance, le savoir passe toujours par l'incorporation d'une manière de voir, d'entendre ou de toucher ${ }^{36}$.

Votre question invite donc à faire un pas supplémentaire pour remettre en cause la manière dont on conçoit l'expertise en histoire de l'art, et à lui adjoindre des pratiques attentionnelles développées en analyse du mouvement. Si l'on songe aux liens que les études labaniennes entretenaient avec les formes de pédagogie expérimentale du Bauhaus $^{37}$, on mesure l'ébranlement que de telles pratiques ne manqueraient pas de susciter dans le champ de l'histoire de l'art, aujourd'hui.

Pour ma part, je mets en œuvre un tel croisement méthodologique dans mes travaux sur les dessins de la chorégraphe américaine Lucinda Childs. Ils ont été décrits par des historiennes de l'art à travers le prisme du minimalisme. En enquêtant sur les pratiques de lecture auxquelles ils donnaient lieu dans le studio de la chorégraphe à New York, au début des années 1970, j'ai constaté que leur déchiffrement nécessitait que le mouvement soit exécuté en même temps qu'il était lu (fig. 11a-b). Cette forme d'attention aux parcours, qui passe du croquis à l'espace du studio dans lequel ils sont exécutés, papier à la main, m'a permis de proposer une bifurcation historiographique majeure. Plutôt que de considérer uniquement les dessins dans le prolongement des pratiques sérielles, je les rapproche, désormais, de l'abstraction géométrique à laquelle a recours la diaspora allemande de New York, et en particulier, la pionnière de la danse moderne Hanya Holm, auprès de qui Lucinda Childs se forme dans les années 1950. Dans l'école de Holm, où les rudiments de la labanotation étaient enseignés, on pratiquait cette forme particulière de lecture réflexive qui permet de passer de la page au geste. Ainsi, bien que les formes graphiques des partitions de Childs diffèrent de celles de la cinétographie Laban, ces deux situations de lecture partagent une forme comparable d'incorporation du mouvement. Une telle enquête invite, donc, à nuancer la coupure généralement admise entre danse moderne et postmoderne. Je n'aurais 
pas pu réaliser cette opération historique sans m'être, moi-même, engagé dans cette forme d'appréhension des tracés. Mon apprentissage de la cinétographie auprès de Noëlle Simonet au Conservatoire de Paris a joué, à cet égard, un rôle décisif, en m’apprenant à lire en dansant.

- Mickaël Bouffard. À la question de savoir si le propre corps de l'historien de l'art peut être un outil supplémentaire à son arsenal méthodologique traditionnel, la réponse est oui. Comme l'expliquait Daniel Jacquet, qui défend la légitimité de la « recherche incorporée » (embodied research) sur le modèle de l'archéologie expérimentale, la « littérature technique sur les savoirs du corps pose déjà une première série de problèmes liés à leur intelligibilité pour un lecteur dont la corporalité et les savoirs gestuels (référentiel ou mémoire kinesthésique) sont éloignés dans le temps des auteurs et du public cible des sources ${ }^{38} »$. Une partie des sous-entendus contenus dans ces sources techniques peut être éclaircie par la mise en pratique. L'historien doit alors se prêter au jeu de l'incorporation, ce qui, dans certaines disciplines comme la danse, nécessite des capacités physiques minimales sans lesquelles les conclusions pourraient être faussées.

Mais je pense qu'il faudra du temps pour rendre légitime dans notre discipline ce genre de démarche.

11a-b. Lucinda Childs, notes de direction pour les danseurs, Melody Excerpt (1977), vers 1981, encre, crayon et ruban adhésif sur papier, recto-verso, $12,6 \times 7,6 \mathrm{~cm}$, Pantin, Centre national de la danse, fonds Lucinda Childs.

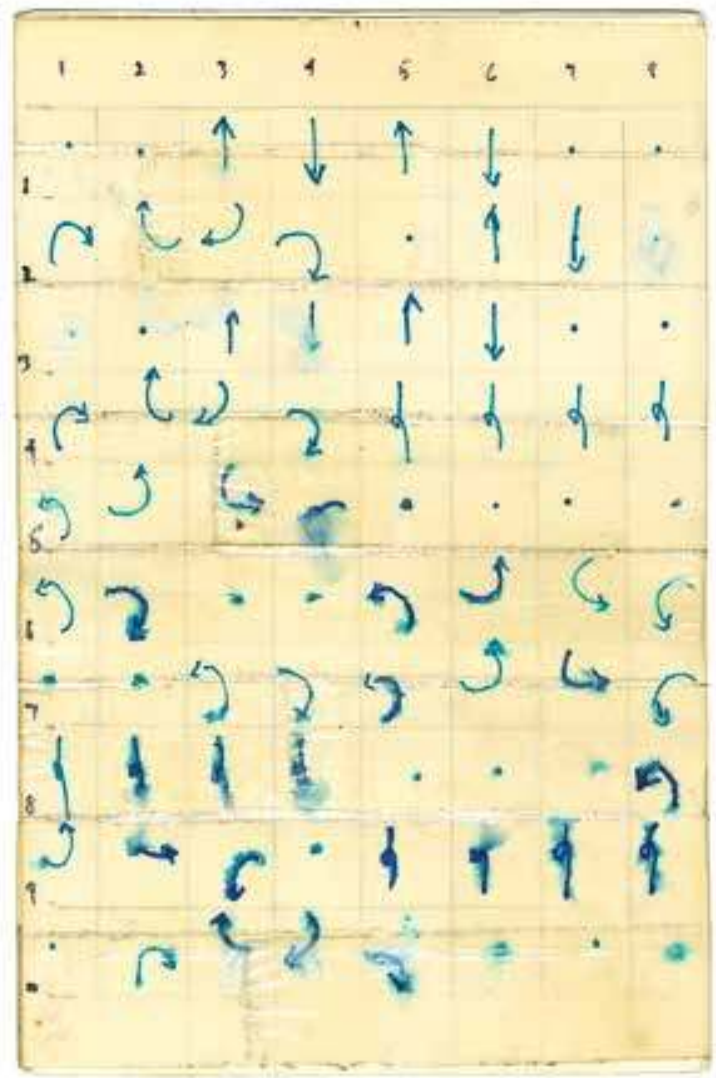
Quand j'ai donné le cours «La Renaissance hors d'Italie » à l'Université de Montréal, j'ai voulu initier mes étudiants à la danse noble de la fin du XVIe siècle, le temps d'une soirée, avec des praticiens spécialistes de cette période. L'objectif était de leur faire prendre

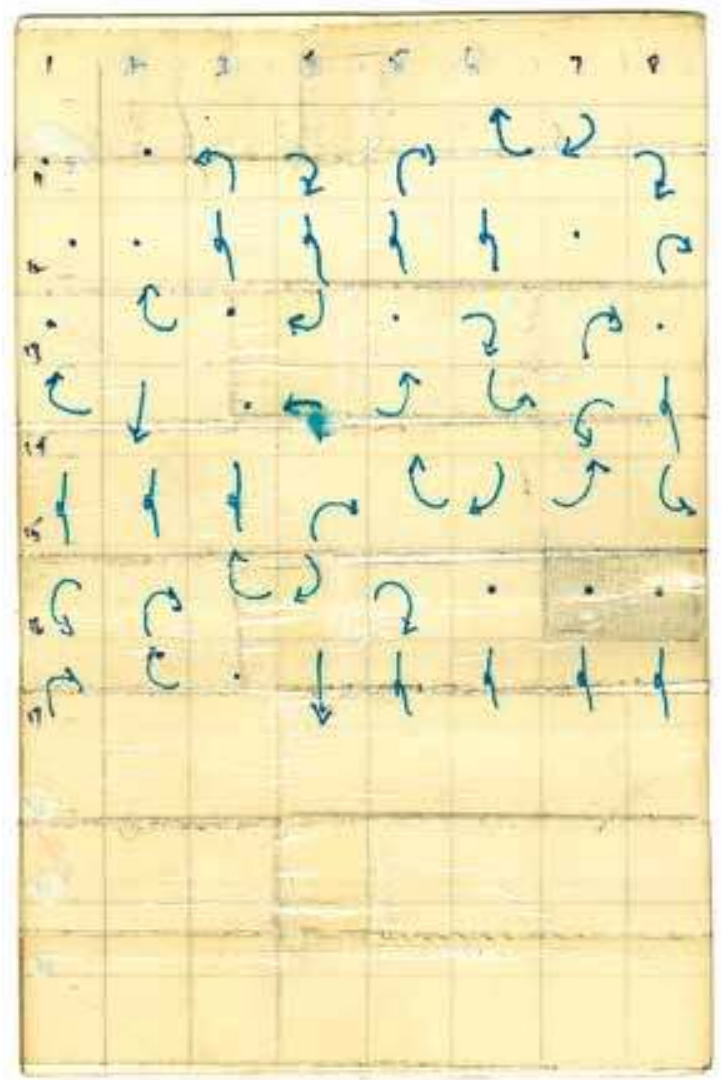


conscience, par un début d'incorporation, du fossé qui séparait la grande mobilité des jambes de l'impassibilité du buste, lieu d'expression de la décence et du décorum du noble danseur. Cette initiation pratique avait suffi à transformer leur regard sur le portrait où l'extrême rigidité du haut du corps prenait tout son sens dans ce genre pictural essentiellement panégyrique. L'activité a néanmoins failli être annulée par le département qui y a vu dans un premier temps une mascarade frivole, inappropriée aux études universitaires (la danse portant toujours les stigmates du discours judéo-chrétien). J'ai finalement obtenu l'autorisation de maintenir l'activité après avoir expliqué la démarche de mon expérience pédagogique qui se voulait une extrapolation des thèses de Michael Baxandall.

En effet, Baxandall l'a montré : le regard est façonné par l'expérience. Ainsi, un chercheur qui incorpore un savoir corporel ancien, même partiellement et avec toutes les précautions épistémologiques nécessaires, verra son regard transformé. En pratiquant la danse ancienne, il ne verra plus les images du danseur de l'époque moderne avec la même naïveté, il évitera probablement certains écueils interprétatifs et élucidera (avec de la persévérance) les passages de certains traités qui demeurent opaques à la seule lecture. Cela lui permet de mieux décoder les appuis, de déterminer à quelle étape du mouvement l'artiste a choisi de représenter son danseur, s'il cherche la grâce ou la caricature, s'il connaît la technique ou non, s'il décrit une pratique contemporaine ou s'il cite l'antique, etc. Je ne dis pas que cette façon de procéder est une méthode miracle, ni qu'elle met l'historien de l'art à l'abri d'anachronismes ou de distorsions. Je dis seulement que l'incorporation de ces données anciennes peut éclairer notre compréhension du passé et qu'elle présente ni plus ni moins de fragilité par rapport au savoir historique que nos méthodes d'enquêtes traditionnelles, si ce n'est qu'elle est plus neuve et donc moins éprouvée. Après de nombreuses années, les méthodes de l'archéologie expérimentale ont fini par faire leurs preuves et ont permis de montrer qu'elles étaient parfois le seul accès à certains pans de la connaissance. II ne reste plus qu'à démontrer que ces méthodes peuvent également porter leurs fruits au sein de l'histoire de l'art.

La contribution de Juliet Bellow

a été traduite de I'anglais par Michèle Veubret. 


\section{Inge Baxmann}

Inge Baxmann est professeure à l'Institut d'études théâtrales de I'Université de Leipzig. De 2001 à 2009, elle était directrice de la Tanzarchiv Leipzig. Elle a publié, entre autres, Social Media New Masses (avec T. Beyes, C. Pias, Chicago, University of Chicago Press 2016) ; Les Archives internationales de la Danse (avec C. Rousier, P. Veroli, Paris, Éditions du CND, 2006) ; Mayas, Pochos und Chicanos: Die transnationale Nation (Munich, Wilhelm Fink, 2007) ; Mythos: Gemeinschaft. Körper und Tanzkulturen in der Moderne (Munich, Wilhelm Fink, 2000).

\section{Juliet Bellow}

Juliet Bellow est professeure associée d'histoire de I'art à l'American University (Washington, DC). Elle a publié Modernism on Stage: The Ballets Russes and the Parisian Avant-Garde (Burlington, Ashgate Press, 2013), et elle a été consultée, en tant que spécialiste, pour l'exposition Diaghilev and the Ballets Russes, 1909-1929: When Art Danced with Music (Washington, National Gallery of Art, 2013). Elle est également l'auteure d'articles parus dans The Art Bulletin, Art Journal, American Art, et Modernism / Modernity, et a contribué à des catalogues d'exposition sur Sonia Delaunay (Londres, Tate Modern / musée d'Art moderne de la Ville de Paris), Merce Cunningham (Minneapolis, Walker Art Center) et Auguste Rodin (Londres, Courtauld Institute of Art / Paris, musée Rodin).

\section{Mickaël Bouffard}

Chargé de recherche au Centre d'Étude de la Langue et des Littératures Françaises (CELLF) de Sorbonne Université, Mickaël Bouffard est historien de l'art, spécialiste de l'iconographie théâtrale, du costume et des pratiques scéniques sous I'Ancien Régime (théâtre, opéra, ballet). Il est présentement codirecteur artistique et scientifique de Théâtre Molière Sorbonne avec Georges Forestier et Jean-Noël Laurenti.

\section{Pauline Chevalier}

Pauline Chevalier est maîtresse de conférences en histoire de l'art à l'université de Bourgogne - Franche-Comté et conseillère scientifique à I'INHA, où elle est à l'origine d'un programme de recherche sur les pratiques graphiques en danse (INHA / BNF / CND). Elle poursuit ses recherches sur les convergences entre littérature, danse, sculpture et image en mouvement dans l'art américain depuis les années 1960 . Elle est notamment I'auteure d'Une Histoire des espaces alternatifs à New York, de SoHo au South Bronx (1969-1985) (Dijon, Les Presses du réel, 2017) et codirectrice de l'ouvrage Le Musée par la scène: le spectacle vivant au musée (avec A. Mouton-Rezzouk et D. Urrutiaguer, Montpellier, Deuxième Époque, 2018).

\section{Lou Forster}

Commissaire d'exposition, historien de l'art et dramaturge, Lou Forster travaille à l'intersection entre la danse et les sciences humaines. À partir de 2014, il lance un projet autour de Lucinda Childs qui prend la forme d'une exposition monographique, de la donation des archives de la chorégraphe au Centre national de la danse et d'un doctorat, en cours, à l'EHESS. Depuis 2018, il participe au programme de recherche "Chorégraphies. Écriture et dessin, signe et image dans les processus de création et de transmission chorégraphiques", en tant que chargé d'études et de recherche à I'INHA. Il est diplômé du premier cycle d'analyse et d'écriture du mouvement en cinétographie Laban au Conservatoire de Paris (2020). En parallèle de ses recherches personnelles, il fonde la plateforme abd avec Lenio Kaklea, pour développer des projets à l'intersection entre danse et théorie critique.

\section{Marie Glon}

Marie Glon est maîtresse de conférences en danse à l'université de Lille et participe activement au programme de recherche de I'INHA «Chorégraphies. Écriture et dessin, signe et image dans les processus de création et de transmission chorégraphiques $\left(\mathrm{XV}^{\mathrm{e}}-\mathrm{XXI}{ }^{\mathrm{e}}\right.$ siècles) ». Elle a été rédactrice en chef de la revue Repères, cahier de danse de 2003 à 2015 et est également l'auteure d'une thèse intitulée Les Lumières chorégraphiques. Les maîtres de danse européens au cœur d'un phénomène éditorial (1700-1760), réalisée sous la direction de Georges Vigarello.

\section{NOTES}

1. "Chorégraphies. Écriture et dessin, signe et image dans les processus de création et de transmission chorégraphiques (XVe- $\mathrm{XXl}^{\mathrm{e}}$ siècles) ", voir la présentation du programme en ligne, sur le site de l'INHA : https://www. inha.fr/fr/recherche/le-departement-des-etudes-et-dela-recherche/domaines-de-recherche/histoire-des-disciplines-et-des-techniques-artistiques/choregraphies.html.

2. Citons, parmi de multiples exemples d'hier et d'aujourd'hui, les travaux actuels de Dominique Brun, qui livre son interprétation d'œuvres phares de la modernité en danse à partir de leurs traces iconographiques ; ceux de Gaëlle Bourges, qui trouvent leur ferment dans une expérience de regard sur des œuvres plastiques ; ou encore les démarches de Pierre Droulers, Gerard \& Kelly...

3. Atelier de recherche avec Marie Glon (université de Lille), Sylviane Pagès (université Paris 8 Vincennes Saint-Denis), Laurent Sebillotte (CND), Juliette Riandey (CND), Pauline Chevalier (INHA), Lou Forster (INHA), Antonin Liatard (INHA) et Dominique Solane, la fille de la chorégraphe. 
4. Francis Haskell, L'historien et les images, Alain Tachet et Louis Évrard (trad. fr.), Paris, Gallimard, 1995, p. 15 [éd. orig. : History and its Images: Art and Representation of the Past, New Haven / Londres, Yale University press, 1993].

5. Deborah Jowitt, dans Time and the Dancing Image, revient sur ce tableau de François Lepaulle, image très souvent reproduite mais finalement peu commentée : "And there's a serious bit of poetic license in Lepaulle's much-copied picture : the sylph is barefoot. Taglioni's slippers were a vital part of the illusion she created". Deborah Jowitt, Time and the Dancing Image, Berkeley / Los Angeles, University of California Press, 1988, p. 29-30.

6. Atelier de recherche mené à l'Opéra avec Mathias Auclair (BNF), Laurent Barré (CND), Pauline Chevalier (INHA), Séverine Forlani (BNF), Lou Forster (INHA), Marie Glon (université de Lille), Bénédicte Jarrasse (aCD, Labex Obvil, université Paris-Sorbonne), Marine Kisiel (musée d'Orsay / INHA), Antonin Liatard (INHA), Bruno Ligore (Université Nice - Côte d'Azur), Julie Ramos (Université Paris 1 - Panthéon Sorbonne). L'atelier a ensuite donné lieu à une journée d'études le 25 janvier 2020.

7. Bruno Ligore (université de Nice), dans son travail sur les dessins de Charles Blasis conservés à Paris, à Milan et à New York, cherche justement à mettre en évidence la somme des références convoquées par Blasis (dessins, sculptures, peintures), qui voyait aussi la scène comme le lieu du musée mis en spectacle et des œuvres vivantes.

8. Le programme de recherche mené à I'INHA proposera également, fin 2021, un guide des sources chorégraphiques à destination des historiens de l'art, cartographie des fonds d'archives conservés dans des institutions consacrées à la danse et aux arts du spectacle et qui présentent un intérêt remarquable pour I'histoire de l'art.

9. Laurence Louppe, Poétique de la danse contemporaine, Bruxelles, Contredanse, 1997, p. 61.

10. Georges Didi-Huberman, L'image survivante, Paris, Les Éditions de Minuit, 2002, p. 256.

11. À propos de l'art de Farhad Ostovani : « Nous sentons bien que l'attention visuelle est doublée, à tous les étages, par une attention motrice. » Jean Starobinski, "Farhad Ostovani ", dans La beauté du monde, Paris, Gallimard, 2016, p. 1130.

12. Bernard Berenson, Les peintres italiens de la Renaissance (1894-1907), Neville Rowley (éd.), John Pope-Hennessy (intro.), Louis Gillet (trad. fr.), Paris, Klincksieck, 2017, p. 144.

13. Gabriele Brandstetter, Poetics of Dance: Body, Image and Space in the Historical Avant-Gardes (1995), Oxford, Oxford University Press, 2015.

14. Juliet Bellow, «Instant et séquence : le paradoxe du simultanisme de Sonia Delaunay ", dans Anne Monfort et Cécile Godefroy (dir.), Sonia Delaunay : les couleurs de l'abstraction, cat. exp. (Paris, musée d'Art moderne de la Ville de Paris / Londres, Tate Modern, 2014-2015), Paris, Paris Musées, 2014, p. 98-102 ; voir aussi Gordon Hughes, «Envisioning Abstraction: The Simultaneity of Robert Delaunay's First Disk », dans The Art Bulletin, 89, n² 2, juin 2007, p. 306-332.
15. Nell Andrew, Moving Modernism: The Urge to Abstraction in Painting, Dance, Cinema, Oxford, Oxford University Press, 2020, p. XVI ; voir aussi Robin Veder, The Living Line: Modern Art and the Economy of Energy, Hanovre, NH, Dartmouth University Press, 2015.

16. Mark Franko, Dancing Modernism / Performing Politics, Bloomington / Indianapolis, Indiana University Press, 1995.

17. En ligne, URL : https://vimeo.com/256861491 (consulté le 6 juillet 2020).

18. Sharon Fermor, "On the Question of Pictorial Evidence for Fifteenth-Century Dance Technique ", dans Dance Research, vol. V, no 2, Automne 1987, p. 18-32.

19. Michael Baxandall, L'œil du Quattrocento : I'usage de la peinture dans I'Italie de la Renaissance, Yvette Delsaut (trad. fr.), Paris, Gallimard, 1985, p. 120-126 [éd. orig. : Painting and Experience in Fifteenth Century Italy: a Primer in the Social History of Pictorial Style, Oxford, Clarendon Press, 1972].

20. Sur la snapshot approach, voir William Smith, "Dance Iconography: Traditions, Techniques and Trends ", dans Thomas Heck et Robert Erenstein (dir.), Picturing Performance: The Iconography of the Performing Arts in Concept and Practice, Rochester, University of Rochester Press, 1999, p. 113-135.

21. Wayne Franits (dir.), Looking at SeventeenthCentury Dutch Art: Realism Reconsidered, Cambridge, Cambridge University Press, 1997.

22. Paris, Archives nationales, $\mathrm{O}^{1} 2984$ : «Mémoire du pain, vin, verres et bouteilles qui ont été fournis aux répétitions du ballet du Triomphe de l'Amour » et "Mémoire du pain, vin, verres et bouteilles qui ont été fournis aux représentations du ballet du Triomphe de l'Amour".

23. Première édition : Laszlo Moholy-Nagy, Malerei, Fotografie, Film, Bauhausbücher, nº 8, Leipzig, Hesse und Becker, 1927, réédité en 1967.

24. "Dessinez-vous avec goût, et naturellement, dans la moindre des poses. Il faut que le danseur puisse, à chaque instant, servir de modèle au peintre et au sculpteur. [...] Dans vos pirouettes observez l'équilibre le plus parfait, et soyez toujours bien placé en les commençant, en les tournant et en les terminant. Arrêtezles avec aplomb et assurance; que le dessin de la position de votre corps, de vos bras, de vos jambes, soit correct, et prononcé avec grâce. » Charles Blasis, Traité élémentaire, théorique et pratique de l'art de la danse, contenant les développements et les démonstrations des principes généraux et particuliers, qui doivent guider le danseur, Milan, chez Joseph Beati et Antoine Tenenti, 1820, p. 23.

25. Coypel, Antoine, Discours prononcés dans les Conférences de l'Académie Royale de Peinture et de Sculpture, Paris, Collombat, 1721, p. 167.

26. Franciscus Lang, s.j., Dissertatio de actione scenica cum figuris eandem explicantibus et observationibus quibusdam de arte comica, Munich, Typis Mariæ Magdalenæ Riedlin viduæ, 1727, réédité : traduit du latin et présenté par Alfred Siemon Golding, New York, Theatre Library Association, 1984, p. 19. 
27. Kellom Tomlinson, The Art of Dancing Explained, Londres, K. Tomlinson, 1724-1735, p. 3.

28. Juliet Bellow et Nell Andrew, « Inventing Abstraction? Modernist Dance in Europe ", dans Stephen Ross et Allana C. Lindgren (dir.), The Modernist World, Londres / New York, Routledge, 2015, p. 329-338.

29. Kate Elswit, Watching Weimar Dance, Oxford, Oxford University Press, 2014, p. xi.

30. Kathy Foley, " Hanako and the European Imagination ", dans Asian Theater Journal, vol. 5, n 1, printemps 1988, p. 76.

31. Juliet Bellow, "Hand Dance: Auguste Rodin's Drawings of the Cambodian Royal Ballet », dans The Art Bulletin, vol. 101, n 3, automne 2019, p. 37-65.

32. Voir notamment Christine Roquet, $v u$ du geste. Interpréter le mouvement dansé, Pantin, Centre national de la danse, 2020.

33. Le projet est décrit par Alexandra Gerstein, « Flight, Arabesque and Cantilever in Rodin's Mouvement Studies ", dans Alexandra Gerstein (dir.), Rodin and Dance: The Essence of Movement, cat. exp. (Londres, Courtauld Institute Gallery, 2016-2017), Londres, Paul Holberton Publishing, 2016, p. 25-39.

34. Leo Steinberg, «Rodin » (1963), dans Other Criteria: Confrontations with Twentieth-Century Art, Londres / Oxford, Oxford University Press, 1972, p. 339.

35. Voir, en particulier, Charles Goodwin, «Professional Vision ", dans American Anthropologist, 96, n 3, 1994, p. 606-633.

36. Des chercheurs et des chercheuses se sont, d'ailleurs, penchés sur les pratiques d'expertise des mondes de I'art comme Franck Leibovici, Henri Michaux : voir (une enquête), Paris, Presses de l'université Paris-Sorbonne, 2014 ; ou Yaël Kreplak, "Artworks in and as Practices. The Relevance of Particulars ", dans Philippe Sormani, Guelfo Carbone et Priska Gisler (dir.), Practicing Art / Science: Experiments in an Emerging Field, Londres / New York, Routledge, 2018.

37. Les études labaniennes regroupent un ensemble de techniques d'analyses du mouvement développées par le chorégraphe, pédagogue et théoricien Rudolf von Laban en Allemagne à partir du milieu des années 1920. Pour une description précise de son approche du mouvement et de ses liens avec le Bauhaus voir, notamment, Vera Maletic, Body-Space-Expression: The Development of Rudolf Laban's Movement and Dance Concepts, Berlin, Mouton de Gruyter, 1987.

38. Daniel Jaquet, "Entre jeux de mains et jeux de mots. Faire l'expérience ou expérimenter les gestes d'après les textes techniques. Reproduire ou répliquer les objets ", dans Daniel Jaquet et Nicolas Baptiste (dir.), Expérimenter le maniement des armes à la fin du Moyen Âge / Experimente zur Waffenhandhabung im Spätmittelalter, Revue d'Histoire Suisse, Basel, Schwabe, no 39,2016 , p. 12. 